\title{
Determination of the tunneling flight time as the reflected phase time
}

\author{
Tom Rivlin $\odot,{ }^{1, *}$ Eli Pollak $\odot,{ }^{1, \dagger}$ and Randall S. Dumont $\odot^{2, \ddagger}$ \\ ${ }^{1}$ Chemical and Biological Physics Department, Weizmann Institute of Science, 76100 Rehovot, Israel \\ ${ }^{2}$ Department of Chemistry and Chemical Biology, McMaster University, Hamilton, Ontario, Canada L8S 4M1
}

(Received 13 September 2020; revised 29 December 2020; accepted 5 January 2021; published 27 January 2021)

\begin{abstract}
Using the time parameter in the time-dependent Schrödinger equation, we study the time of flight for a particle tunneling through a square barrier potential. Comparing the mean and variance of the energy and the flight time for transmitted and reflected particles, using both density and flux distributions, we find that, when accounting for momentum filtering, the suitably normalized transmitted and reflected distributions are identical in both the density and flux cases. In contrast to previous studies, we demonstrate that these results do not imply a vanishing tunneling time, but rather that the time it takes to tunnel through a square barrier is precisely given by the reflected phase time. For wide barriers, this becomes independent of the barrier width, as predicted independently by MacColl and Hartman. We show that these conclusions can be reached using a variety of arguments, including purely quantum mechanical ones. Analysis of the shapes of the distributions under consideration reveals that wave-packet reshaping is not an explanation for the MacColl-Hartman effect. The results presented here have direct implications for understanding recent experimental results in the study of the barrier crossing of rubidium atoms. The finite width of an incident wave packet significantly "masks" the tunneling time, and induces substantial asymmetry between the flight times of transmitted and reflected atoms.
\end{abstract}

DOI: 10.1103/PhysRevA.103.012225

\section{INTRODUCTION}

It is well known that a quantum mechanical object can pass through a barrier it would be forbidden to traverse classically. This is known as quantum tunneling. However, one significant property of this transition is considered contentious among contemporary researchers [1-5]: How long does this traversal take? This question is related to discussions concerning a phenomenon known as the Hartman effect [6,7], which describes the surprising revelation that the time taken to traverse a barrier appears to be independent of the length of the barrier when said barrier is sufficiently broad. Although controversial, this effect has support experimentally and theoretically $[1,7]$, and even in the relativistic limit [8-10].

On the experimental side, many experiments in the 1990s and early 2000s studied optical analogs of tunneling systems and verified that times independent of barrier lengths could be obtained [11-13], and several experiments since have found that the effect is also valid for quantum systems such as Larmor precession [14-16] and attoclock experiments [14,1720].

There remain unresolved controversies in the experimental results, however, with some claiming that the tunneling time is exactly zero $[18,19,21,22]$, and others claiming that it is small but finite $[11,15,16]$. Both sides have theoretical and computational results that support their claims, and most agree that it is independent of the length of a sufficiently broad barrier.

\footnotetext{
*tom.rivlin@weizmann.ac.il

†eli.pollak@weizmann.ac.il

‡umontr@mcmaster.ca
}

The controversy has recently been renewed thanks in part to recent experimental results measuring the "Larmor time" for barrier crossings involving rubidium atoms $[15,16]$. This is in agreement with the original claim by Hartman, and arguably the earlier claim by MacColl [23]. Others do, however, claim that the measures of tunneling time that lead to the "MacColl-Hartman" effect are associated with lifetimes such as energy decay times $[5,24]$ but not with physical transit times.

This paper contributes to this discussion by demonstrating three main points. The first point is that in the limit of the incident wave packet having a narrow width in momentum space, the contribution from tunneling to the flight time for a square barrier is given by the reflected phase time. This time is finite and independent of width for sufficiently long barriers, and for a symmetric square barrier, differs from the transmitted phase time. The second point is that this demonstrates that the Hartman effect can be observed for flight times, leaving open the possibility of experimentally verifying it. We note that prior to the present results, the Hartman effect has only been studied theoretically for monochromatic waves, and not for wave packets. The final point is that the Hartman effect is not a result of the effects of momentum filtering and wave-packet distortion, as claimed by some $[25,26]$, as we show that the effect persists when these are removed and the transmission is restricted to strictly below-barrier momentum components.

Instead of attempting to define a time operator, we consider the time of flight of the particle as determined by the time parameter appearing in the time-dependent Schrödinger equation. Initially, the particle is described via a Gaussian wave packet whose incident mean kinetic energy is below the potential barrier height, and whose momentum variance is sufficiently small to ensure that above-barrier components of 
the wave packet are much smaller than the mean transmission probability (no "above-barrier transmission"). At the same time the packet is initiated sufficiently far to the left of the barrier to ensure that the initial probability of finding the particle to the right of the barrier is negligible compared to the initial normalization of unity (no "pretransmission"). With these conditions, transmission occurs predominantly through tunneling and any nontunneling contributions may be ignored.

Since time-of-flight experiments typically measure the flux of particles impinging on a detector, we study here both the density and the flux time-of-flight distribution, rather than just the density, as studied previously in Ref. [27]. This also has the advantage that both the mean and the variance of the flight time are well defined, as the time flux distribution at large times goes as $t^{-4}$, in comparison with the density which goes as $t^{-3}[7,28]$.

We generate results both using purely quantum mechanical computations, and with classical trajectories from a classical Wigner dynamics approximation to the quantum mechanics. With the Wigner dynamics results, we presuppose zero tunneling time, and then examine how the overall time of flight compares to the results calculated using full quantum mechanical methods.

In addition to the mean and variance, another aspect of the distributions, their peaks, is studied. Comparisons between the means and peaks of the time-of-flight distributions are made, facilitating comparisons to free particles, and allowing for further explorations of how the wave packet is reshaped by the tunneling process.

For the systems we study, we show that the tunneling process induces minimal changes to the form of the final transmitted wave packet itself: the wave packet retains its Gaussian-like form and its width in time is directly related to its initial spatial width. The main reshaping of the transmitted wave packet is the lowering of its amplitude due to the small transmission amplitude associated with tunneling. We further emphasize this finding with an examination of the variances of the transmitted time and energy distributions, especially in reference to the energy-time uncertainty relation, and make comparisons to analytic results generated with the steepestdescent approximation.

These results negate previous arguments based on wavepacket reshaping which feature in many explanations of the Hartman effect. Our results show, in agreement with Ref. [5], but in contrast to some claims in the literature [25,26], that wave-packet reshaping cannot be invoked as an explanation for the Hartman effect.

Determining the time of flight and the related tunneling time should be achieved without resort to classical or semiclassical mechanics [11,29-31]. Hence, using only the time-dependent Schrödinger equation, we evaluate the mean flight time of transmitted and reflected particles and, in agreement with recent studies [27], find that in the limit that the momentum width of the incident wave packet vanishes, the two are identical. Taking the momentum width to zero provides a bridge between the theoretical definition of the phase time, valid for monochromatic wave packets, and more realistic tunneling wave packets with finite widths.

However, this does not "prove" that the tunneling time vanishes. When we analyze the mean transmission time separately, we find that in the limit of the momentum width of the incident wave packet vanishing it implies a tunneling time which is accurately predicted by the reflected phase time.

This last conclusion is based not only on a comparison of the peak time, that is, the time associated with the peak of the transmitted wave packet as compared to that of a free particle, but also by comparing the mean transmission time for differing barrier heights. We find that the dependence of the mean transmission time on the barrier height is well accounted for by the phase time. A comparison of the mean flight time with classical Wigner computations further strengthens the claim that the tunneling time is given accurately by the phase time.

It should be stressed that this conclusion, that the tunneling time is given by the reflected phase time, is meaningful only for a square barrier and for an incident particle with vanishing momentum variation. Partly this is because in the case of a square barrier, all the "action" occurs in the tunneling region, whereas for a smooth barrier such as a Gaussian-shaped one, the particle is slowed down in the classically allowed region. The phase time includes both this slowing down and the speeding up due to tunneling.

The situation is further complicated when the momentum width of the incident particle is finite. Not only must one consider the well-understood phenomenon of momentum filtering [27,32], but in addition the width induces large asymmetry between the mean transmitted and reflected flight times. There is also the possibility that contributions from momentum components close to and higher than the barrier height can drown out both the Hartman effect and the effects of momentum filtering. The implications of these observations to understanding the recent Larmor time experimental results $[15,16]$ on the scattering of rubidium atoms on a Gaussian barrier are considered here.

In Sec. II we review briefly the theory needed for implementing the computations. Results are presented in Sec. III and we end with a Discussion, paying special attention to the implications of this work on the aforementioned rubidium Larmor time experiments.

\section{THEORY}

We consider a one-dimensional Gaussian wave packet with a large width in space (or, equivalently, a small width in momentum), which is initially placed far to the left of a square potential barrier with an incident mean momentum in the positive direction, that is, towards the barrier. The initial position, initial momentum, and their distributions are all chosen such that the initial probabilities of the wave packet being found to the right of the barrier in position space and above it in momentum space are both negligible. The Hamiltonian operator for this system is

$$
\hat{H}=\frac{\hat{p}^{2}}{2 M}+V(\hat{q})
$$

for a particle with mass $M$, where the potential $V(\hat{q})$ is given by

$$
V(\hat{q})=V^{\ddagger}[\Theta(\hat{q}+a)-\Theta(\hat{q}-a)]
$$


for the Heaviside function $\Theta(\hat{q})$, barrier height $V^{\ddagger}$, and barrier width $2 a$ centered around the origin (we restrict our analysis here to symmetric barriers).

The initial wave packet is given by

$$
\begin{aligned}
& \Psi(q, t=0) \\
& \quad=\langle q \mid \Psi\rangle=\left(\frac{\Gamma}{\pi}\right)^{\frac{1}{4}} \exp \left(-\frac{\Gamma}{2}(q-x)^{2}+\frac{i}{\hbar} p_{x}(q-x)\right),
\end{aligned}
$$

where $\Gamma$ is the width parameter, $x$ is the initial mean position of the wave packet, and $p_{x}$ is the initial mean momentum. $x$ is chosen such that $x+\frac{1}{\sqrt{\Gamma}}$ is far more negative than $-a$, and $p_{x}$ is chosen such that $\left(\frac{p_{x}^{2}}{2 M}+\frac{\hbar^{2} \Gamma}{M}\right) \ll V^{\ddagger}$.

The time evolution of the initial wave packet was computed by integrating it over all $k=\frac{p}{\hbar}$ when multiplied by $\exp \left(-i \frac{\hbar k^{2}}{2 m} t\right)$. The particular method for doing this for a Gaussian wave packet interacting with a square barrier was based on the method outlined by Papadopoulos [33] (see also [32]).

\section{A. Transition path time distributions}

A transition path time probability distribution [27,34] is defined for observing the particle density evolved to positive time $t: \rho(y, t)=|\Psi(y, t)|^{2}$ at the spatial point $y$ (the "screen"). The transition path time probability distribution (TPTD) for the density is given by [27]

$$
P_{\Psi, \rho}(y, t)=\frac{|\Psi(y, t)|^{2}}{\int_{0}^{\infty} d t|\Psi(y, t)|^{2}} .
$$

There are some implicit assumptions in this definition that must be stressed: it is assumed that the integral in the denominator does not diverge, that the density is well defined at the point $y$, that there are no contributions to the integral from negative times (unlike the presence time distribution [35], which does include negative times), and that the density is positive-valued everywhere (it is a positive-operator-valued measure, or POVM [36]).

Most importantly, we have assumed the existence of a clock that is able to tick once at $t=0$ when the state is prepared, and once again at some time $t$ when the particle is detected at the screen position $y$. This allows $t$ to be a variable that measures the time of flight of the particle. This assumption is made for all the definitions discussed in this work.

A different version of the time distribution of Eq. (4) may be defined for the flux instead of the density. The expression for the flux density $J(q, t)$ at the screen $y$ and time $t$ is well known (the prime denotes the derivative with respect to the coordinate $q$ ):

$$
J(y, t)=\frac{\hbar}{M} \operatorname{Im}\left(\Psi^{*}(y, t) \Psi^{\prime}(y, t)\right) .
$$

Hence, the TPTD for the flux at a given $y$ and $t$ can be expressed as

$$
P_{\Psi, J}(y, t)=\frac{J(y, t)}{\int_{0}^{\infty} d t J(y, t)} .
$$

Useful metrics for analyzing the tunneling time without resorting to classical or semiclassical mechanics can be obtained by considering $P_{\Psi, \rho}$ and $P_{\Psi, J}$ for the reflected and transmitted parts of the density and flux, respectively. This is achieved by considering their values at two points: one far to the right of the barrier such that $y \gg a$, and one far to the left of the initial position such that $-y \ll x$ (the two points are equidistant from the center of the barrier). That ensures that only reflected components of the density or flux are present at $-y$, and only transmitted components at $y$.

This also ensures that $P_{\Psi, J}$ can be used as a POVM, despite the fact that it can have negative values. For the systems being considered here, we are only interested in the value of $P_{\Psi, J}$ far to the left or right of the barrier, where the flux is either entirely negative or entirely positive, such that the resulting flux time distribution is positive and this is well defined. This construction was also used in our recent study of the TPTD for a relativistic electron scattered on a square barrier [10].

Labeling the density TPTD to the right of the barrier at $y$ as $P_{\Psi, \rho, T}$, the density TPTD to the left of the barrier at $-y$ as $P_{\Psi, \rho, R}$, and the flux equivalents of those two quantities as $P_{\Psi, J, T}$ and $P_{\Psi, J, R}$, respectively, we can define the means of all four distributions:

$$
\langle t\rangle_{(\rho, J),(T, R)}=\int_{0}^{\infty} d t t P_{\Psi,(\rho, J),(T, R)} .
$$

Unlike in the case of a free particle, this integral does not diverge when considering both the density and the flux reflected and transmitted time distributions. Note that all four distributions $P_{\Psi,(\rho, J),(T, R)}$ are separately normalized to unity: the fact that the transmission probability is far smaller than the reflection probability is not important for this part of the analysis. Also note that this integral is only over the interval $[0, \infty]$, as with the definitions in Eqs. (4) and (6).

One of the advantages of considering the flux time distributions is that due to its $t^{-4}$ dependence at long times, one may also obtain the second moments of the time distribution and the associated variances $\left(\sigma_{t}\right)_{J,(T, R)}^{2}$ :

$$
\left\langle t^{2}\right\rangle_{J,(T, R)}=\int_{0}^{\infty} d t t^{2} P_{\Psi, J,(T, R)}
$$

and

$$
\left(\sigma_{t}\right)_{(\rho, J),(T, R)}^{2}=\left\langle t^{2}\right\rangle_{J,(T, R)}-\langle t\rangle_{J,(T, R)}^{2} .
$$

This is not possible for the density time distribution, as it goes as $t^{-3}$ at long times such that the second moment diverges (although in practice the density time distribution's variance was approximated numerically and found to be very similar to the flux's variance).

One of the difficulties in examining the tunneling time is that for a finite width of the incident wave packet one has to account for the effect of momentum filtering [27,32]. Due to tunneling and the related exponential dependence of the transmission probability on the incident momentum of the wave packet, higher momenta will be preferentially transmitted. This leads to significant differences in the mean transmitted and reflected times. The filtering effect will become smaller as the momentum width of the incident wave packet becomes smaller. Therefore, we are interested in the dependence of the TPTDs on the width parameter $\Gamma$ and the difference between the reflected and transmitted mean times for the two operators 
$\rho$ and $J$ :

$$
\Delta t_{(\rho, J)}(\Gamma)=\langle t\rangle_{(\rho, J), R}(\Gamma)-\langle t\rangle_{(\rho, J), T}(\Gamma) .
$$

In the limit of vanishing momentum width of the incident wave packet, the filtering effect will vanish and any remaining difference should reflect the tunneling time. Hence, we define the tunneling flight time difference as

$$
t_{\mathrm{TFT},(\rho, J)}=-\lim _{\Gamma \rightarrow 0} \Delta t_{(\rho, J)}(\Gamma) .
$$

Further discussion of the implications of this definition for the density time distribution may be found in Ref. [27]. These implications also carry over to the flux case.

In addition to the time differences, the mean times themselves $\left(\langle t\rangle_{\rho, R},\langle t\rangle_{J, R},\langle t\rangle_{\rho, T}\right.$, and $\left.\langle t\rangle_{J, T}\right)$ can be separately considered in the $\Gamma \rightarrow 0$ limit. These quantities will also form the basis of other useful metrics for the numerical studies presented in the next section.

\section{B. Free particles}

One way used by many [37] to understand the tunneling time is to compare the transmitted particle's time distribution at the screen to that of a free particle. One propagates the initial wave packet defined as in Eq. (3) with the free-particle time propagator $\exp \left(-i \frac{\hbar k^{2}}{2 M} t\right)$ onto the screen point $y$ and studies the resulting distribution of density or flux arrival times.

There is, however, a formal difficulty when considering a free particle's time distribution. Unlike the transmitted particle, for a free-particle wave function $\Psi_{\mathrm{FP}}(y, t)$ the integral $\int d t t\left|\Psi_{\mathrm{FP}}(y, t)\right|^{2}$ diverges [28]. The same is also true of the equivalent integral for the flux. Time averages of the transmitted time distributions for the density and flux are undefined in the free-particle case and so cannot be compared to those of the transmitted particle.

The accepted alternative is to compare instead the peak times, or the times at which the density and flux at the screen are maximal. Taking the $\Gamma \rightarrow 0$ limit helps in this case, too, as it makes the peak and mean times for the transmitted particle as close as possible, as the results section will show. Thus, one useful tunneling time metric can be written as

$$
t_{\operatorname{maxdiff},(\rho, J)}=\lim _{\Gamma \rightarrow 0}\left[t_{\max ,(\rho, J), \mathrm{T}}(\Gamma)-t_{\max ,(\rho, J), \mathrm{FP}}(\Gamma)\right]
$$

for the density and flux peaks of the free particle $t_{\text {max },(\rho, J), \mathrm{FP}}(\Gamma)$ and the tunneled particle $t_{\max ,(\rho, J), \mathrm{T}}(\Gamma)$.

In the $\Gamma \rightarrow 0$ limit, the peak of the time distribution of the free-particle wave packet matches the simple classical time of flight of the free particle. For a free-particle wave packet initially peaked at $q=x$, the distance to the screen is $y+|x|$, and thus the classical time of flight is this distance divided by the speed $\frac{p_{x}}{M}$. By the same logic, the time a classical particle would spend traversing the same length as the barrier width, which one might call the "naive" barrier traversal time, is given by $\frac{2 a M}{p_{x}}$.

Hence, a barrier traversal time $t_{\text {trav }}$ can be defined as follows:

$$
t_{(\rho, J), \text { trav }}=t_{\max ,(\rho, J), \mathrm{T}}-\frac{M(y+|x|-2 a)}{p_{x}} .
$$

The latter term on the right can be thought of as the $\Gamma \rightarrow 0$ limit free-particle peak time of flight, minus the naive barrier traversal time. This leaves only a metric for how long the free particle spent outside the barrier region: the free-particle "nonbarrier" traversal time. Subtracting this from the peak time of flight of the transmitted particle gives a measure of how long the transmitted particle spent inside the barrier: a barrier traversal time.

\section{Phase time}

It is an open question whether the MacColl-Hartman effect implies a zero tunneling time or a fixed, finite time. The phase time (sometimes called the Wigner time [38]) is believed to be involved if the latter is true. There is a phase time associated with both the transmitted and reflected parts of the distribution. If the probability of transmission as a function of $k$ is given by $|T(k)|^{2}$, and the probability of reflection by $|R(k)|^{2}$, then the phase times $\Phi_{T}(k)$ and $\Phi_{R}(k)$ are defined as $\frac{M}{\hbar k}$ times the derivative with respect to $k$ of the arguments, or phases, of $T(k)=|T(k)| \exp \left[i \varphi_{T}(k)\right]$ and $R(k)=|R(k)| \exp \left[i \varphi_{R}(k)\right]$, respectively [1]:

$$
\Phi_{T}(k)=\frac{M}{\hbar k} \frac{d \varphi_{T}}{d k}, \quad \Phi_{R}(k)=\frac{M}{\hbar k} \frac{d \varphi_{R}}{d k} .
$$

The quantities $T(k)$ and $R(k)$ for the symmetric square barrier are given by [32]

$$
T(k)=\frac{2 i k K \exp (-2 i k a)}{\left(k^{2}+K^{2}\right) \sin (2 K a)+2 i k K \cos (2 K a)}
$$

and

$$
R(k)=\frac{\left(k^{2}-K^{2}\right) \sin (2 K a)}{\left(k^{2}+K^{2}\right) \sin (2 K a)+2 i k K \cos (2 K a)},
$$

where

$$
K=\sqrt{k^{2}-\frac{2 M V^{\ddagger}}{\hbar^{2}}} .
$$

The phase times can be computed by noting that

$$
\frac{d \varphi_{T}}{d k}=\operatorname{Im}\left(\frac{d \ln T}{d k}\right)
$$

and similar for $R(k)$. In principle, the phase times as defined here shed light on the tunneling time for monochromatic scattering only. We will demonstrate that they also give a good approximation to tunneling flight times for narrowin-momentum wave packets whose incident energies are sufficiently below the barrier height.

For the square barrier, the following relationship holds for all values of $a, p_{x}$, and $V^{\ddagger}$ :

$$
\Phi_{R}\left(\frac{p_{x}}{\hbar}\right)-\Phi_{T}\left(\frac{p_{x}}{\hbar}\right)=\frac{2 a M}{p_{x}} .
$$

The term on the right-hand side is the naive barrier traversal time and will be important when analyzing the implications of the phase times for the tunneling times. This relationship, which depends on the width but not the height of the barrier, is an exception to the rule that symmetric barriers have equal transmitted and reflected phase times in the monochromatic limit. 
When considering the scattering of an incident (nonmonochromatic) wave packet, the transmission probability for the wave packet is obtained by averaging over the momentum components. The resulting averaged transmission and reflection probabilities, denoted as $\left|T_{\Psi}\right|^{2}$ and $\left|R_{\Psi}\right|^{2}$, respectively, are given by

$$
\left|T_{\Psi}\right|^{2}=\frac{1}{\sqrt{\Gamma \pi}} \int_{-\infty}^{\infty} d k|T(k)|^{2} \exp \left(-\frac{\left(k-k_{\text {init }}\right)^{2}}{\Gamma}\right)
$$

for transmission, and an equivalent expression is used to obtain the total reflection probability $\left|R_{\Psi}\right|^{2}$. Here, $k_{\text {init }}$ is the mean wave number of the incident wave packet. These integrals also serve as the normalization constants for the energy distributions. Similarly, equivalent integrals for the normalization of the transmitted and reflected flux $\left|T_{\Psi, J}\right|^{2}$ and $\left|R_{\Psi, J}\right|^{2}$ are given by multiplying the integrand of Eq. (20) and its reflection equivalent by $\hbar k$. The normalization factors for the free-particle density and flux energy distributions are given by removing $\left|T_{\Psi}\right|^{2}$ from the integrand, and multiplying by $\hbar k$ in the flux case.

The dependence of the transmission and reflection probabilities on the wave number $k$ is the cause of the momentum filtering phenomenon discussed above. Especially in the deep tunneling regime, the transmission probability $|T(k)|^{2}$ increases exponentially with increasing $k$. This means that higher- $k$ components will be preferentially included in the transmitted wave packet, as compared to the reflected wave packet where the reflection coefficient is almost unity in this regime and hardly changes. This effect implies that the transmitted wave packet will travel faster than the reflected one and tends to mask any short-time contribution due to tunneling. By taking the limit of $\Delta t$ as $\Gamma \rightarrow 0$, it is possible to remove the effects of momentum filtering since there is in principle only one momentum component at $\Gamma=0$.

\section{Wave-packet energies}

In addition to defining the means and variances in time of the four time-of-flight distributions for the transmitted particles, it is also possible to define their means and variances in energy. This is useful not only for checking the energy-time uncertainty of the wave packets, but also the extent to which the scattering reshapes the wave packet since, initially, the wave packets are minimum uncertainty states.

Due to the difficulty in defining the time operator, one does not have a time-energy analog to the momentum-position uncertainty principle. However, as shown in [28], the relation

$$
\left(\sigma_{E}\right)^{2}\left(\sigma_{t}\right)^{2} \geqslant \frac{\hbar^{2}}{4}
$$

holds if one defines the energy variance of the density using weak values.

The procedure for defining this quantity is as follows: the time-dependent Schrödinger equation for the wave packet at time $t$ is

$$
\hat{H}\left|\Psi_{t}\right\rangle=i \hbar \frac{\partial}{\partial t}\left|\Psi_{t}\right\rangle
$$

By considering the weak-value time mean of the Hamiltonian operator

$$
\begin{aligned}
\langle H(q)\rangle_{\rho(T, R)}= & \frac{1}{\int_{0}^{\infty} d t\left\langle\Psi_{t} \mid q\right\rangle\left\langle q \mid \Psi_{t}\right\rangle} \\
& \times \int_{0}^{\infty} d t\left\langle\Psi_{t} \mid q\right\rangle\left\langle q \mid \Psi_{t}\right\rangle \frac{\left\langle q|\hat{H}| \Psi_{t}\right\rangle}{\left\langle q \mid \Psi_{t}\right\rangle},
\end{aligned}
$$

one obtains the expressions

$$
\begin{aligned}
\langle E\rangle_{\rho(T, R)}= & \frac{i \hbar}{\int_{0}^{\infty} d t\left|\Psi_{(T, R)}(q, t)\right|^{2}} \\
& \times \int_{0}^{\infty} d t \Psi_{(T, R)}^{*}(q, t) \frac{\partial \Psi_{(T, R)}(q, t)}{\partial t}
\end{aligned}
$$

and

$$
\left\langle E^{2}\right\rangle_{\rho(T, R)}=\frac{\hbar^{2}}{\int_{0}^{\infty} d t\left|\Psi_{(T, R)}(q, t)\right|^{2}} \int_{0}^{\infty} d t\left|\frac{\partial \Psi_{(T, R)}(q, t)}{\partial t}\right|^{2} .
$$

The equivalent expressions for the flux $J(y, t)$ are not given in [28], but can be shown to have similar forms to the expressions given there:

$$
\langle E\rangle_{J(T, R)}=\frac{i \hbar}{\int_{0}^{\infty} d t J(q, t)} \int_{0}^{\infty} d t \frac{J(q, t)}{\Psi_{(T, R)}(q, t)} \frac{\partial \Psi_{(T, R)}(q, t)}{\partial t}
$$

and

$$
\begin{aligned}
\left\langle E^{2}\right\rangle_{J(T, R)}= & \frac{\hbar^{2}}{\int_{0}^{\infty} d t J(q, t)} \\
& \times \int_{0}^{\infty} d t \frac{J(q, t)}{\left|\Psi_{(T, R)}(q, t)\right|^{2}}\left|\frac{\partial \Psi_{(T, R)}(q, t)}{\partial t}\right|^{2} .
\end{aligned}
$$

In all of these expressions, the variable $q$ is set to the screen location $+y$ for the transmitted quantities and $-y$ for the reflected quantities. The variances can then be defined using

$$
\left(\sigma_{E}\right)_{(\rho, J),(T, R)}^{2}=\left\langle E^{2}\right\rangle_{(\rho, J),(T, R)}-\langle E\rangle_{(\rho, J),(T, R)}^{2} .
$$

When the energy variances $\left(\sigma_{E}\right)_{J,(T, R)}^{2}$ defined here are multiplied by the respective time variances $\left(\sigma_{t}\right)_{J,(T, R)}^{2}$, the products are greater than $\frac{\hbar^{2}}{4}$, as is the case for the density time distribution. This is discussed in more detail in Appendix A.

\section{E. Steepest-descent approximation}

The steepest-descent approximation can be used to provide a convenient, accurate approximation to the time-evolving wave packet beyond the barrier. This can then be used to obtain analytic expressions for the energy and time variances and their product, and be used to assess the effects of wave-packet reshaping.

The calculation begins with the energy representation of the time-evolving wave packet. The steepest-descent approximation eliminates the associated integral over $k$ by pulling out the principal contribution arising from the neighborhood of the saddle-point wave number $k^{\sharp}$. Using this expression, we find the most probable tunneling time, the time $t_{\mathrm{mp}}$, for which the wave packet has greatest amplitude. Expanding the exponent of the wave packet to second order about the most 
probable time gives an explicit Gaussian form for the timeevolving wave packet. This expression affords straightforward computation of the time and weak-value energy moments of interest in this paper. A more detailed derivation is given in Appendix B, and the main results are provided here.

The weak-value energy moments, in this approximation, are

$$
\langle E\rangle_{\rho, T}=E_{\mathrm{mp}}^{\sharp},
$$

where $E_{\mathrm{mp}}^{\#}$ is the energy corresponding to $k_{\mathrm{mp}}^{\#}$, the saddlepoint wave number evaluated at the peak time $t_{\mathrm{mp}}$ (expressions for which are given in Appendix B), and

$$
\left\langle E^{2}\right\rangle_{\rho, T}=E_{\mathrm{mp}}^{\sharp 2}+\frac{1}{2} \hbar^{2}\left[\operatorname{Re} \eta+(\operatorname{Im} \eta)^{2}(\operatorname{Re} \eta)^{-1}\right],
$$

where $\eta / 2$ is the complex coefficient of $\left(t-t_{\mathrm{mp}}\right)^{2}$ in the explicit Gaussian approximation to the time-evolving wave packet (an expression for $\eta$ is given in Appendix B). The steepest-descent variance of the weak-value energy of the transmitted wave packet thus has the form

$$
\sigma_{E, \rho, T}^{2}=\frac{1}{2} \hbar^{2}\left[\operatorname{Re} \eta+(\operatorname{Im} \eta)^{2}(\operatorname{Re} \eta)^{-1}\right] .
$$

The approximate time moments are

$$
\langle t\rangle_{\rho, T}=t_{\mathrm{mp}}
$$

and

$$
\left\langle t^{2}\right\rangle_{\rho, T}=t_{\mathrm{mp}}^{2}+\frac{1}{2}(\operatorname{Re} \eta)^{-1} .
$$

The resulting variances are

$$
\sigma_{t, \rho, T}^{2}=\frac{1}{2}(\operatorname{Re} \eta)^{-1}
$$

and

$$
\sigma_{E, \rho, T}^{2} \sigma_{t, \rho, T}^{2}=\frac{1}{4} \hbar^{2}\left[1+\left(\frac{\operatorname{Im} \eta}{\operatorname{Re} \eta}\right)^{2}\right]
$$

The real and imaginary parts of $\eta$ have the following leading terms, for small $\Gamma$ :

$$
\operatorname{Re} \eta \sim \Gamma v_{\mathrm{mp}}^{\sharp 2}
$$

and

$$
\operatorname{Im} \eta \sim \Gamma^{2} v_{\mathrm{mp}}^{\sharp 2}\left[\left(\frac{d^{2} \varphi_{T}}{d k^{2}}\right)_{\mathrm{mp}}^{\sharp}-\frac{\hbar t_{\mathrm{mp}}}{m}\right],
$$

where the $\sharp$ superscript and mp subscript represent evaluating the derivative at $k=k_{\mathrm{mp}}^{\sharp}$.

Therefore, for $v_{\mathrm{mp}}^{\sharp}=\frac{\hbar}{m} k_{\mathrm{mp}}^{\sharp}$ for small $\Gamma$,

$$
\begin{aligned}
& \sigma_{E, \rho, T}^{2} \sim \frac{\Gamma}{2} \hbar^{2} v_{\mathrm{mp}}^{\sharp 2}, \\
& \sigma_{t, \rho, T}^{2} \sim \frac{\Gamma^{-1}}{2} v_{\mathrm{mp}}^{\sharp-2},
\end{aligned}
$$

and

$$
\sigma_{E, \rho, T}^{2} \sigma_{t, \rho, T}^{2} \sim \frac{1}{4} \hbar^{2}\left\{1+\Gamma^{2}\left[\left(\frac{d^{2} \varphi_{T}}{d k^{2}}\right)_{\mathrm{mp}}^{\sharp}-\frac{\hbar t_{\mathrm{mp}}}{m}\right]^{2}\right\} .
$$

The flux-weighted moments are the same as the densityweighted moments in the steepest-descent approximation, as the velocity factor of the flux weighting is treated as a prefactor to the exponential function in the energy representation integrand. It does not affect the saddle point, and it cancels out when moments are computed.

\section{F. Classical Wigner dynamics}

One way to gain insight into the tunneling time is by comparing the quantum results with an analogous approximate theory based on classical trajectories which fully accounts for momentum filtering. For this purpose we use a classical Wigner dynamics formulation [39-44]. The classical Wigner approximation for the TFT was computed in both the density and flux cases and compared to the numerically exact quantum equivalents.

The Wigner function formalism is a phase-space-based representation of quantum mechanics that permits the existence of classical-mechanics-like quantities such as probability distributions (more formally "quasidistributions") over position and momentum. The Wigner function for the density is defined as the Weyl transformation of the density operator $\hat{\rho}$ (divided by $2 \pi \hbar$ ), and the Wigner function for the flux operator is defined in an equivalent way. In both cases, in this work they were then time evolved using classical mechanical free-particle time propagation, and integrated over all $k$ to obtain functions that are analogous to $\Psi(q, t)$.

For a free particle, the position-space Wigner representation of the density of a Gaussian wave packet $\Psi(q, t)$ is given by

$$
\begin{aligned}
w_{\Psi, \rho}(q, t)= & \frac{1}{\pi \hbar} \int_{-\infty}^{\infty} d k \exp \left[-\Gamma\left(q-x-\frac{\hbar k}{M} t\right)^{2}\right. \\
& \left.-\frac{1}{\hbar^{2} \Gamma}\left(\hbar k-\hbar k_{\text {init }}\right)^{2}\right]
\end{aligned}
$$

where $x$ and $k_{\text {init }}$ are the initial position and wave-number peaks, respectively. The Wigner representation of the flux is the same as for the density, except with the integrand multiplied by an extra $\frac{\hbar k}{M}$ factor.

In the classical Wigner approximation, one assumes classical time evolution. For the square barrier, this implies free-particle motion, as in Eq. (41), except for in the barrier region. We assume, as in [27], that a trajectory hitting the barrier with momentum $\hbar k$ is reflected from it with probability $|R(k)|^{2}$ and transmitted instantaneously to the right edge of the barrier with probability $|T(k)|^{2}$. This implies that the time distributions measured at the screen locations $y$ and $-y$ are obtained by multiplying the integrand in Eq. (41) by $|T(k)|^{2}$ and $|R(k)|^{2}$, respectively. The instantaneous barrier traversal time is accounted for by subtracting the barrier width $2 a$ from the path traversed by the transmitted particle, thereby assuming the time spent inside the barrier is zero. Taking the difference between the flight time calculated using quantum and Wigner dynamics defined in this way will enable us to understand how momentum filtering affects the flight time.

This classical Wigner dynamics approximation accounts for momentum filtering since the transmitted and reflected time distributions at the respective screen locations $y$ and $-y$ are weighted by the transmission and reflection probabilities. We do note that with this procedure we implicitly assume that the momentum is filtered throughout the trajectory, i.e., before and after the barrier. 

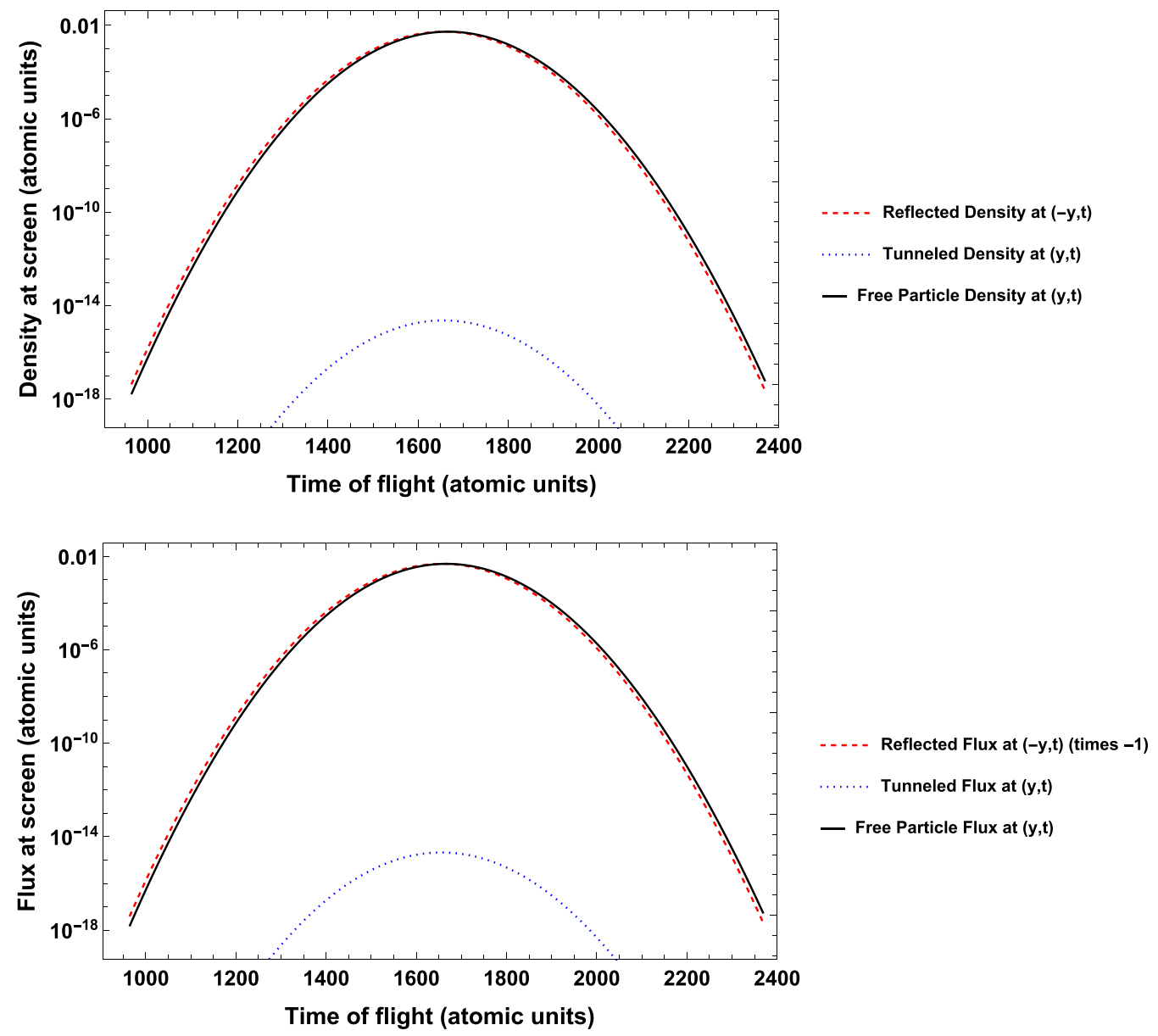

FIG. 1. Quantum mechanical time density and flux distributions. The initial position $x=-500$, the initial momentum $p_{x}=0.9$, the width parameter $\Gamma=0.00009, y=1000, a=5, V^{\ddagger}=1.5$. The reflected flux is multiplied by -1 for the sake of comparison to the other distributions. Note the logarithmic scale used for the distributions.

The normalized density and flux distributions are then obtained as in Eqs. (4) and (6), respectively. The time moments were then obtained as in Eqs. (7) and (8).

\section{RESULTS}

The results for the reflected and tunneled wave packets were obtained following the propagation method set out by Papadopoulos [33]. All of the results were obtained by numerically integrating the appropriate quantities using Mathematica version 12.1's "double exponential" integration method. Atomic units were used throughout. Convergence testing revealed the numerical integration techniques used were stable to 10 significant figures.

For comparisons between the numerically exact quantum mechanical (QM) and approximate Wigner dynamics computations, a quantity $\Omega$ was defined as in Ref. [27] to quantify the squared differences between them, such that for $N$ comparisons between times measured quantum mechanically and using Wigner dynamics (with each $N$ representing a different value of $\Gamma$ ),

$$
\Omega=\frac{1}{N} \sum_{j=1}^{N} \frac{\left(t_{\mathrm{QM}, j}-t_{W, j}\right)^{2}}{t_{\mathrm{QM}, j}^{2}} .
$$

Six time distributions were examined and compared for each value of the width parameter $\Gamma$. In each case, we were interested in the evolution of a wave packet that begins as a Gaussian centered around the point $x<0$ with momentum centered around $p_{x}$, and with width parameter $\Gamma$. This wave packet was then either propagated freely to a screen at the point $y$, or it tunneled through a square barrier centered around $q=0$ before arriving at the screen at $y$, or it was reflected by that barrier and measured at a screen at the point $-y$. In each case, the density and flux distributions at the screen were computed as a function of the time of flight. The distributions were generated using either numerically exact quantum mechanical or approximate Wigner dynamics. The six unnormalized quantum mechanical distributions for a given value of $\Gamma$ are shown in Fig. 1. When $a=5, V^{\ddagger}=1.5$ and $p_{x}=0.9$ as in the distributions in the figure, the transmission probability is $4.4 \times 10^{-13}$.

\section{A. Tunneling flight time}

When the six distributions are normalized as in Eqs. (4) and (6), such that the reflected distributions are divided by $\left|R_{\Psi}\right|^{2}$, the transmitted distributions are divided by $\left|T_{\Psi}\right|^{2}$, and the free-particle distributions divided by 1 , the reflected and transmitted distributions are very close to each other. The 


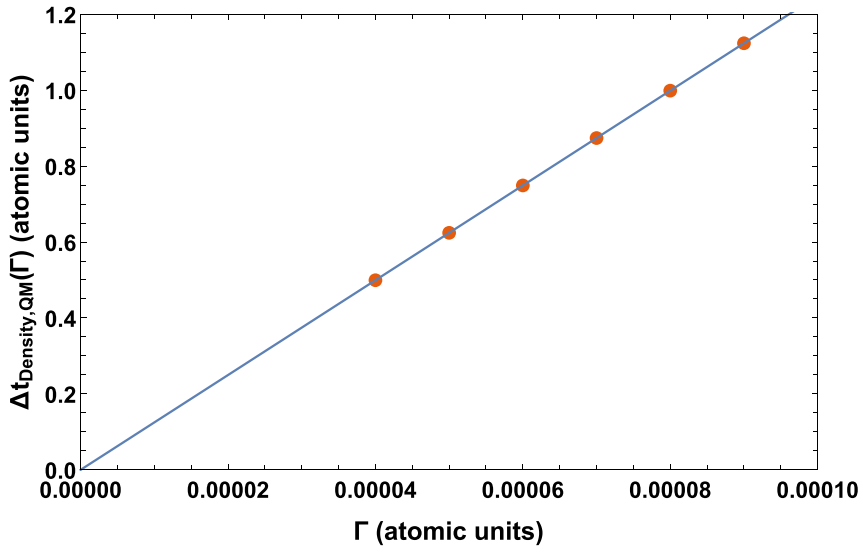

(a)

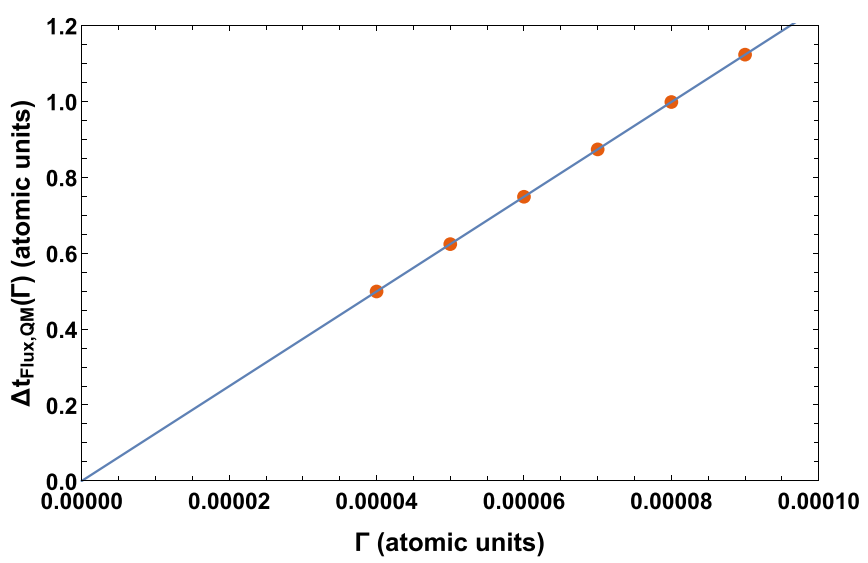

(c)

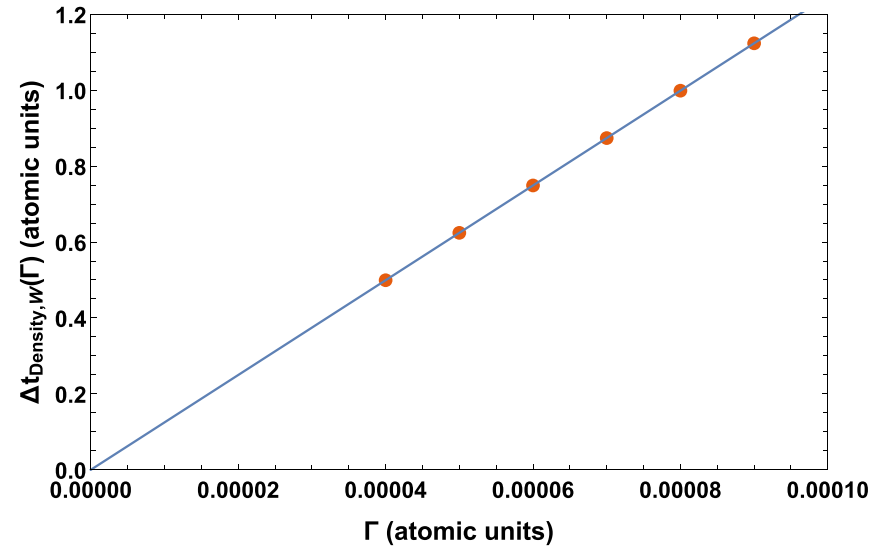

(b)

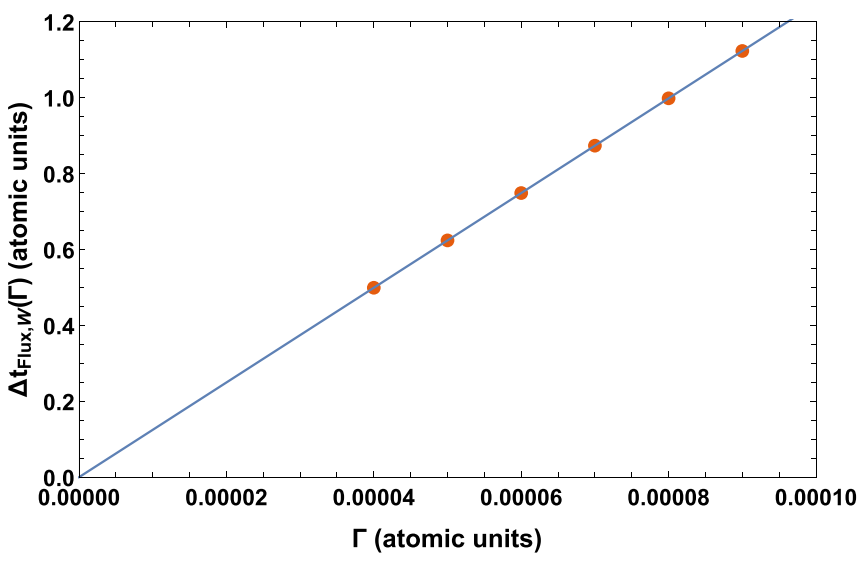

(d)

FIG. 2. The differences between the reflected and transmitted mean time of flight for the density [upper panels: (a) $\Delta t(\Gamma)$ for the numerically exact quantum density time distribution, (b) $\Delta t(\Gamma)$ for the classical Wigner density time distribution] and flux [lower panels: (c) $\Delta t(\Gamma)$ for the numerically exact quantum flux time distribution, (d) $\Delta t(\Gamma)$ for the classical Wigner flux time distribution] time distributions using numerically exact quantum dynamics (left panels) and classical Wigner dynamics (right panels). The non- $\Gamma$ parameters used are as in Fig. 1. In each plot, the points represent the results of the calculations, and the lines are lines of best fit through those points, the fitting parameters of which are shown in Table I.

extent to which they are not identical depends on the Gaussian spatial width parameter $\Gamma$, as shown in Fig. 2.

This figure illustrates the calculation of the tunneling flight time (TFT), which is defined as the limit as $\Gamma \rightarrow 0$ of the

TABLE I. Tunneling flight times for the density $\left(t_{\mathrm{TFT}, \rho}\right)$ and flux $\left(t_{\mathrm{TFT}, J}\right)$ time distributions using numerically exact quantum propagation and classical Wigner dynamics, and $\alpha_{(\rho, J),(\mathrm{QM}, W)}$, the slopes of the fits used to produce them. The errors from the QM-Wigner comparisons for both the density and the flux $\Omega_{(\rho, J)}$ [as in Eq. (42)] are also shown. Results are presented for four different sets of representative physical parameters.

\begin{tabular}{|c|c|c|c|c|c|c|c|c|c|c|}
\hline Parameters & $t_{\mathrm{TFT}, \rho, \mathrm{QM}}$ & $t_{\mathrm{TFT}, \rho, \mathrm{W}}$ & $t_{\mathrm{TFT}, \mathrm{J}, \mathrm{QM}}$ & $t_{\mathrm{TFT}, J, W}$ & $\alpha_{\rho, \mathrm{QM}}$ & $\alpha_{\rho, W}$ & $\alpha_{J, \mathrm{QM}}$ & $\alpha_{J, W}$ & $\Omega_{\rho}$ & $\Omega_{J}$ \\
\hline$p_{x}=0.9$ & & & & & & & & & & \\
\hline $\begin{array}{l}a=5 \\
V^{\ddagger}=1.5\end{array}$ & $-1.3131 \times 10^{-4}$ & $-1.3135 \times 10^{-4}$ & $-6.9452 \times 10^{-5}$ & $-6.9550 \times 10^{-5}$ & $1.2487 \times 10^{4}$ & $1.2479 \times 10^{4}$ & $1.2485 \times 10^{4}$ & $1.2477 \times 10^{4}$ & $1.1418 \times 10^{-3}$ & $1.1418 \times 10^{-3}$ \\
\hline $\begin{array}{l}p_{x}=0.4 \\
a=5 \\
V^{\ddagger}=1.5\end{array}$ & $-1.4819 \times 10^{-3}$ & $-1.4807 \times 10^{-3}$ & $1.6472 \times 10^{-4}$ & $1.6458 \times 10^{-4}$ & $4.4156 \times 10^{4}$ & $4.4122 \times 10^{4}$ & $4.4101 \times 10^{4}$ & $4.4068 \times 10^{4}$ & $1.5016 \times 10^{-3}$ & $1.5015 \times 10^{-3}$ \\
\hline $\begin{array}{l}p_{x}=0.9 \\
a=1 \\
V^{\ddagger}=1.5\end{array}$ & $-2.7038 \times 10^{-5}$ & $-2.7023 \times 10^{-5}$ & $-4.8829 \times 10^{-7}$ & $-4.9450 \times 10^{-7}$ & $3.5594 \times 10^{3}$ & $3.5573 \times 10^{3}$ & $3.5585 \times 10^{3}$ & $3.5564 \times 10^{3}$ & $1.1580 \times 10^{-3}$ & $1.1580 \times 10^{-3}$ \\
\hline $\begin{array}{l}p_{x}=0.9 \\
a=5 \\
V^{\ddagger}=2.0\end{array}$ & $-7.7904 \times 10^{-5}$ & $-7.7827 \times 10^{-5}$ & $-2.0478 \times 10^{-5}$ & $-2.0567 \times 10^{-5}$ & $1.0803 \times 10^{4}$ & $1.0797 \times 10^{4}$ & $1.0801 \times 10^{4}$ & $1.0795 \times 10^{4}$ & $1.1202 \times 10^{-3}$ & $1.1204 \times 10^{-3}$ \\
\hline
\end{tabular}


difference in mean times between the reflected and transmitted wave packets. The TFT is presented for both the density and the flux. The calculation has been performed using exact quantum and approximate Wigner dynamics computations. As the figure shows, in all cases the difference between the reflected and transmitted particles' mean times of flight linearly tends to zero as the width parameter is reduced. The largest mean time difference recorded on the graph is approximately 1 , whereas the peak times of the reflected and transmitted distributions at the screen occur at approximately 1600 time units. Clearly, the reflected and transmitted distributions peak at almost the exact same time when $\Gamma$ is small. The TFT itself was calculated by fitting the points to a straight line and calculating its intercept.

The values of $\Gamma$ used to calculate the TFT were chosen such that the wave packet was as narrow in energy as possible with a negligible probability of finding the particle to the right of the barrier at $t=0$. The initial distance of $x=-500$ distance units allowed for the computation of results for $\Gamma$ as low as $4 \times 10^{-5}$. For $\Gamma=3 \times 10^{-5}$, there was a nontrivial probability, approximately $4 \times 10^{-5}$, of the wave packet being located to the right of the barrier at $t=0$, which notably impacted the results. This was not the case for the $\Gamma=4 \times 10^{-5}$ results shown in Fig. 2, where the probability was approximately $3 \times 10^{-6}$. Over the range studied, increasing $\Gamma$ by $1 \times 10^{-5}$ decreased the probability of the wave packet being located to the right of the barrier at $t=0$ by a factor of approximately 10 .

The actual values of the four tunneling flight times, calculated from the results in Fig. 2 in both the quantum and Wigner function cases and for both the density and the flux, are presented in Table I. The $\Delta t_{(\rho, J),(\mathrm{QM}, W)}(\Gamma)$ values were fit to lines with intercepts $t_{\mathrm{TFT}(\rho, J),(\mathrm{QM},) W}$ and slopes $\alpha_{(\rho, J),(\mathrm{QM}, W) \text {. The }}$ error $\Omega$ for the comparisons between quantum and Wigner results for both the density and the flux are also provided. The transmission probabilities for each of the four sets of parameters considered in Table I were $4.4 \times 10^{-13}, 1.9 \times 10^{-15}$, $8.4 \times 10^{-3}$, and $7.9 \times 10^{-16}$, respectively.

The errors in the fits used to generate the tunneling flight times $t_{\mathrm{TFT},(\rho, J),(\mathrm{QM}, W)}$ are not shown in Table I, but are lower than the values of $\Omega$. For instance, for the first set of parameters in the table, $t_{\mathrm{TFT}, \rho \text {, } \mathrm{QM}}$ was fitted to its value with a standard error of $1.0403 \times 10^{-5}$. Similar standard errors were obtained for the other quantities and parameters. These standard errors are slightly larger than the numerical errors found in convergence testing. The standard error is small enough to make the TFT significantly different from zero, though it is not anticipated that this has any physical significance. Preliminary testing with nonlinear fits have shown that the TFT can be made to be smaller than the standard error when a cubic fit is used, although again we do not expect this to have substantial physical significance.

It is clear that once the effects of momentum filtering are removed by narrowing the initial wave packet down, the reflected and transmitted wave packets' time-of-flight distributions are identical, and the TFT is negligible in all four cases. However much time the tunneled particle spends in the barrier region, it is clear that, for this system, the reflected particle can be interpreted as spending the same amount of time in that region.

\section{B. Classical Wigner dynamics mean transmission times}

Comparing the slopes in Table I reveals that $\Delta t(\Gamma)$ is similar for both the density and the flux operators for both quantum and Wigner dynamics. The mean time of flight for the transmitted particle can be compared directly in the Wigner and quantum cases. Since it was presupposed that the tunneling time was zero when producing results using the Wigner dynamics approximations, the difference in mean time of flight between these two distributions can be interpreted as a measure of the tunneling time.

Table II and Figure 3 are key to understanding this section. They both show the difference in mean time of flight as the width $\Gamma \rightarrow 0$. From these results it becomes clear that, under the interpretation of the difference between the two different means as a representation of the tunneling time, the tunneling time is precisely given by the reflected phase time. This is true regardless of the initial parameters, as long as the initial momentum distribution is well below the barrier height and the wave packet has a negligible amplitude to the right of the barrier at $t=0$. This result will be reinforced in the subsequent sections, which focus on the quantum dynamics, rather than the Wigner dynamics.

\section{Variances}

Here, we demonstrate that, apart from momentum filtering, the only change that tunneling through the square barrier causes to the shape of the wave packet is in its absolute amplitude. We do so by examining the variances of the energy and time distributions of the transmitted and reflected wave packets.

Figure 4 shows the energy and time variances for the transmitted density distribution, along with their product, as functions of $\Gamma, \Gamma^{-1}$, and $\Gamma^{2}$, respectively. The points were calculated using the weak-value time averages as outlined in Ref. [28] and as described in Sec. II and Appendix A. The lines were produced by fitting data points generated using the steepest-descent approximations outlined in Sec. II and Appendix B (with numerical methods used to solve the equation generating $k_{\mathrm{mp}}^{\sharp}$ ).

As predicted by the steepest-descent approximation, Eqs. (38), (39), and (40), we find that the energy variance tends linearly towards zero as $\Gamma \rightarrow 0$, the time variance tends linearly towards zero as $\Gamma^{-1} \rightarrow 0$, and the product tends linearly towards $\frac{\hbar^{2}}{4}$ from above as $\Gamma^{2} \rightarrow 0$ (recalling that $\hbar=1$ in our units).

Along with the results for the transmitted density distribution, equivalent results for the other distributions of Fig. 1 were also generated, and found to be similar, tending in the same way to the same asymptotes. These results demonstrate that the transmitted wave packet is still a minimum uncertainty state, up to the small corrections introduced by the momentum-filtering effects of the barrier. This shows that the narrow-in-momentum, below-barrier wave packets considered here are not distorted by the tunneling process. In view of this, wave-packet reshaping cannot be invoked as an explanation of the MacColl-Hartman effect. 
TABLE II. Mean of the tunneling flight time distribution using QM $\left(\langle t\rangle_{\rho, \mathrm{QM}}\right)$ and Wigner dynamics $\left(\langle t\rangle_{\rho, W}\right)$, compared to the reflected phase time $\Phi_{R}\left(\frac{p_{x}}{\hbar}\right)$. Similar results were obtained for the flux distributions, with a relative error of $3.736 \times 10^{-10}$ for the first set of parameters, for instance. The last column on the right indicates the very high accuracy of the reflected phase time as the measure of the tunneling time.

\begin{tabular}{lcccc}
\hline \hline Parameters & $\langle t\rangle_{\rho, W}$ & $\langle t\rangle_{\rho, \mathrm{QM}}$ & $\Phi_{R}\left(\frac{p_{x}}{\hbar}\right)$ & $\sqrt{\frac{\left\{\langle t\rangle_{\rho, \mathrm{QM}}-\left[\langle t\rangle_{\rho, W}+\Phi_{R}\left(\frac{p_{x}}{\hbar}\right)\right]\right\}^{2}}{\left(\langle t\rangle_{\rho, \mathrm{QM}}\right)^{2}}}$ \\
\hline$p_{x}=0.9, a=5, V^{\ddagger}=1.5$ & 1655.556 & 1657.057 & 1.501634 & $2.299 \times 10^{-10}$ \\
$p_{x}=0.4, a=5, V^{\ddagger}=1.5$ & 3725.000 & 3727.967 & 2.966954 & $2.293 \times 10^{-10}$ \\
$p_{x}=0.9, a=1, V^{\ddagger}=1.5$ & 1664.444 & 1665.947 & 1.502891 & $6.963 \times 10^{-11}$ \\
$p_{x}=0.9, a=5, V^{\ddagger}=2.0$ & 1655.556 & 1656.800 & 1.24421 & $3.501 \times 10^{-10}$ \\
\hline \hline
\end{tabular}

\section{Other measures}

Thus far, we have appealed to comparisons to classical Wigner dynamics to argue that the tunneling time is given by the phase time. Here, we present other arguments in favor of this assertion. The first of these arguments is based on comparisons to free particles with identical initial distributions. This will be especially useful when analyzing recent experimental results, as will be seen in the Discussion section.

As discussed above, one difficulty with comparisons to free particles is that the mean of the free-particle time distributions diverges. It is therefore necessary to focus on the peak of the free particle's time distribution instead, for both the density and the flux. It was anticipated that taking the $\Gamma \rightarrow 0$ limit would also cause the peak of the tunneled particle's time-of-flight distribution $t_{\max ,(\rho, J), T}$ to coincide with its mean $\langle t\rangle_{(\rho, J), T}$, due to the number of momentum components being minimized. Table III displays these quantities for $\Gamma \rightarrow 0$, for different sets of physical parameters, using numerically exact quantum mechanical propagation.

The results in this table confirm that, for both the density and the flux operators and a square barrier, the difference be- tween the mean and peak locations becomes negligibly small as $\Gamma$ tends to zero. This suggests that the location of the peak of the transmitted distribution is still a useful measure of the time of flight, and allows for comparisons to the free-particle distributions' peaks.

With this in mind, Table IV displays comparisons between the location of the peak of the transmitted particle's time distribution and the location of the peak of the free particle's time distribution for both the density and the flux in the limit $\Gamma \rightarrow 0$. Also displayed in this table is the transmitted phase time $\Phi_{T}\left(\frac{p_{x}}{\hbar}\right)$, which coincides numerically with $t_{\text {maxdiff, }(\rho, J)}$, as defined in Eq. (12). (The error parameters for the flux are not shown, but are similar to the error parameters for the density.)

In other words, the transmitted particle, defined using either the density or flux time distributions, arrives at the screen earlier than the free particle, and the time difference between the transmitted particle's peak arriving and the free particle's peak arriving is given by $\Phi_{T}\left(\frac{p_{x}}{\hbar}\right)$. As Eq. (19) states, this is equal to $\Phi_{R}\left(\frac{p_{x}}{\hbar}\right)$ minus the free particle's peak barrier traversal time $\frac{2 a M}{p_{x}}$, suggesting that $\Phi_{R}\left(\frac{p_{x}}{\hbar}\right)$ represents the barrier traversal time of the transmitted particle.

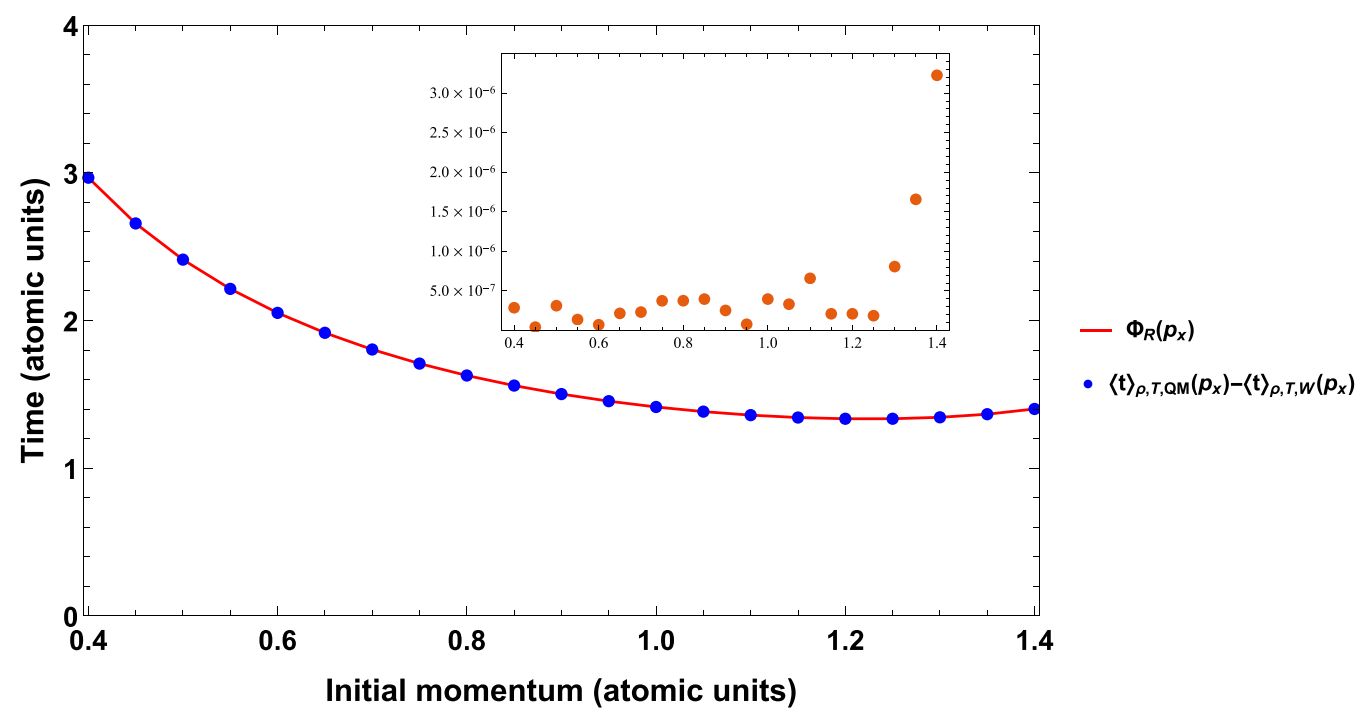

FIG. 3. The difference between the mean time of flight for the density of the tunneled particle propagated quantum mechanically $\langle t\rangle_{\rho, T, \mathrm{QM}}$ and the mean time of flight for the classical Wigner-propagated density of the tunneled particle $\langle t\rangle_{\rho, T, \mathrm{~W}}$ as a function of the initial momentum $p_{x}$ (dots), compared with the reflected phase time $\Phi_{R}$, also as a function of $p_{x}$ (line). The numerical parameters were $a=5, V^{\ddagger}=1.5$, and $\Gamma \rightarrow 0$. Inset: the relative error $\sqrt{\frac{\left[\left(\langle t\rangle_{\mathrm{QM}}\left(p_{x}\right)-\langle t\rangle_{W}\left(p_{x}\right)\right)-\Phi_{R}\left(p_{x}\right)\right]^{2}}{\left(\Phi_{R}\left(p_{x}\right)\right)^{2}}}$ between the points and the curve as a function of $p_{x}$. The agreement between the two very different computations indicates that the tunneling time is given by the reflected phase time. 
TABLE III. The location of the peak of the density distribution $\left(t_{\max , \rho, T}\right)$ and flux distribution $\left(t_{\max , J, T}\right)$ for a transmitted particle, the mean of the distribution for the transmitted density $\left(\langle t\rangle_{\rho, T}\right)$ and the transmitted flux $\left(\langle t\rangle_{J, T}\right)$, and comparisons between these means and peak locations. All quantities are shown in the limit $\Gamma \rightarrow 0$. Results for four different sets of parameters are shown.

\begin{tabular}{|c|c|c|c|c|c|c|}
\hline Parameters & $\langle t\rangle_{\rho, T}$ & $t_{\max , \rho, \mathrm{T}}$ & $\sqrt{\frac{\left(\langle t\rangle_{\rho, T}-t_{\max , \rho, T}\right)^{2}}{\left(\langle t\rangle_{\rho, T}\right)^{2}}}$ & $\langle t\rangle_{J, T}$ & $t_{\max , J, T}$ & $\sqrt{\frac{\left(\langle t\rangle_{J, T}-t_{\max , J, T}\right)^{2}}{\left(\langle t\rangle_{J, T}\right)^{2}}}$ \\
\hline $\begin{array}{l}p_{x}=0.9 \\
a=5 \\
V^{\ddagger}=1.5\end{array}$ & 1657.1 & 1657.1 & $5.3770 \times 10^{-7}$ & 1657.1 & 1657.1 & $7.6642 \times 10^{-8}$ \\
\hline $\begin{array}{l}p_{x}=0.4 \\
a=5 \\
V^{\ddagger}=1.5\end{array}$ & 3728.0 & 3728.0 & $1.3796 \times 10^{-6}$ & 3728.0 & 3728.0 & $1.2535 \times 10^{-6}$ \\
\hline $\begin{array}{l}p_{x}=0.9 \\
a=1 \\
V^{\ddagger}=1.5\end{array}$ & 1665.9 & 1665.9 & $4.9281 \times 10^{-7}$ & 1665.9 & 1665.9 & $5.3423 \times 10^{-8}$ \\
\hline $\begin{array}{l}p_{x}=0.9 \\
a=5 \\
V^{\ddagger}=2.0\end{array}$ & 1656.8 & 1656.8 & $2.7094 \times 10^{-6}$ & 1656.8 & 1656.8 & $5.6736 \times 10^{-8}$ \\
\hline
\end{tabular}

The quantity $t_{\text {maxdiff }}$ also demonstrates that for the square barrier studied here, the transmitted particle takes less time to arrive at the screen than a free particle would, and that the speedup is related to properties of the barrier as measured by the phase time. This analysis is similar to the one performed in a recent paper of ours which studied tunneling with the Dirac equation [10].

Similarly, it can also be shown that $\Phi_{T}$ is related to the difference between the peaks of the free wave packet and the reflected one in the same way that the free and transmitted wave packets are related. The only caveat is that the reflected flux is negative, and thus its trough, not its peak, must be compared to the peak of the free wave packet.

Table IV's error parameter shows good agreement between the time difference and the phase time, but it is not quite as strong as in other results in this work, largely due to numerical error in the precise location of the peak. We found that the results and the errors were relatively unchanged even when altering the comparison such that the filtered momentum was used to calculate $t_{\max , \mathrm{FP}}$. This is in contrast to the figure shown in the Discussion section, where introducing the free-particle time with a filtered momentum has significant effects.

The difference in peak times is also clearly seen in Fig. 5. When the distributions are all normalized to their respective peak values, the shift is visible. In this particular instance, with $\Gamma=9 \times 10^{-5}$ as in Fig. 1, the difference between the times of the peaks is approximately 10 time units. This can be compared to Fig. 2, which shows that at $\Gamma=9 \times 10^{-5}$, the difference between the transmitted and reflected mean times is approximately 1 , which implies that momentum filtering is not the main cause of the shift seen in Fig. 5. The unnormalized peak values themselves are, of course, in actuality many orders of magnitude different from each other due to the small tunneling probability.

While the results of Table IV show that the flight time of the tunneling particle is less than the free-particle time, they do not directly answer the question of how much time the particle spends under the barrier. The results presented in

TABLE IV. The location of the peak of the density distribution $\left(t_{\max , \rho, \mathrm{FP}}\right)$ and flux distribution $\left(t_{\max , J, \mathrm{FP}}\right)$ for a free particle, the same two quantities for the transmitted distribution $\left(t_{\max , \rho, T}, t_{\max , J, T}\right)$, the transmitted particle's phase time $\Phi_{T}\left(\frac{p_{x}}{\hbar}\right)$, and the differences between the transmitted and free particles' peak locations $\left(t_{\operatorname{maxdiff},(\rho, J)}\right)$. All quantities are shown as $\Gamma \rightarrow 0$. Results for four different sets of parameters are shown.

\begin{tabular}{|c|c|c|c|c|c|c|c|c|}
\hline Parameters & $t_{\max , \rho, \mathrm{FP}}$ & $t_{\max , \rho, T}$ & $t_{\mathrm{max}, J, \mathrm{FP}}$ & $t_{\max , J, T}$ & $\Phi_{T}\left(\frac{p_{x}}{\hbar}\right)$ & $t_{\text {maxdiff }, \rho}$ & $t_{\text {maxdiff }, J}$ & $\sqrt{\frac{\left[t_{\max , \rho, \mathrm{FP}}-\Phi_{T}\left(\frac{p_{x}}{\hbar}\right)\right]^{2}}{\left[\Phi_{T}\left(\frac{p_{x}}{\hbar}\right)\right]^{2}}}$ \\
\hline $\begin{array}{l}p_{x}=0.9 \\
a=5 \\
V^{\ddagger}=1.5\end{array}$ & 1666.7 & 1657.1 & 1666.7 & 1657.1 & -9.6095 & -9.6102 & -9.6095 & $7.8725 \times 10^{-5}$ \\
\hline $\begin{array}{l}p_{x}=0.4 \\
a=5 \\
V^{\ddagger}=1.5\end{array}$ & 3750.0 & 3728.0 & 3749.9 & 3728.0 & -22.033 & -22.028 & -21.956 & $2.4313 \times 10^{-4}$ \\
\hline $\begin{array}{l}p_{x}=0.9 \\
a=1 \\
V^{\ddagger}=1.5\end{array}$ & 1666.7 & 1665.9 & 1666.7 & 1665.9 & -0.71933 & -0.71848 & -0.71941 & $1.1182 \times 10^{-3}$ \\
\hline $\begin{array}{l}p_{x}=0.9 \\
a=5 \\
V^{\ddagger}=2.0\end{array}$ & 1666.7 & 1656.8 & 1666.7 & 1656.8 & -9.8669 & -9.8713 & -9.8670 & $4.4679 \times 10^{-4}$ \\
\hline
\end{tabular}



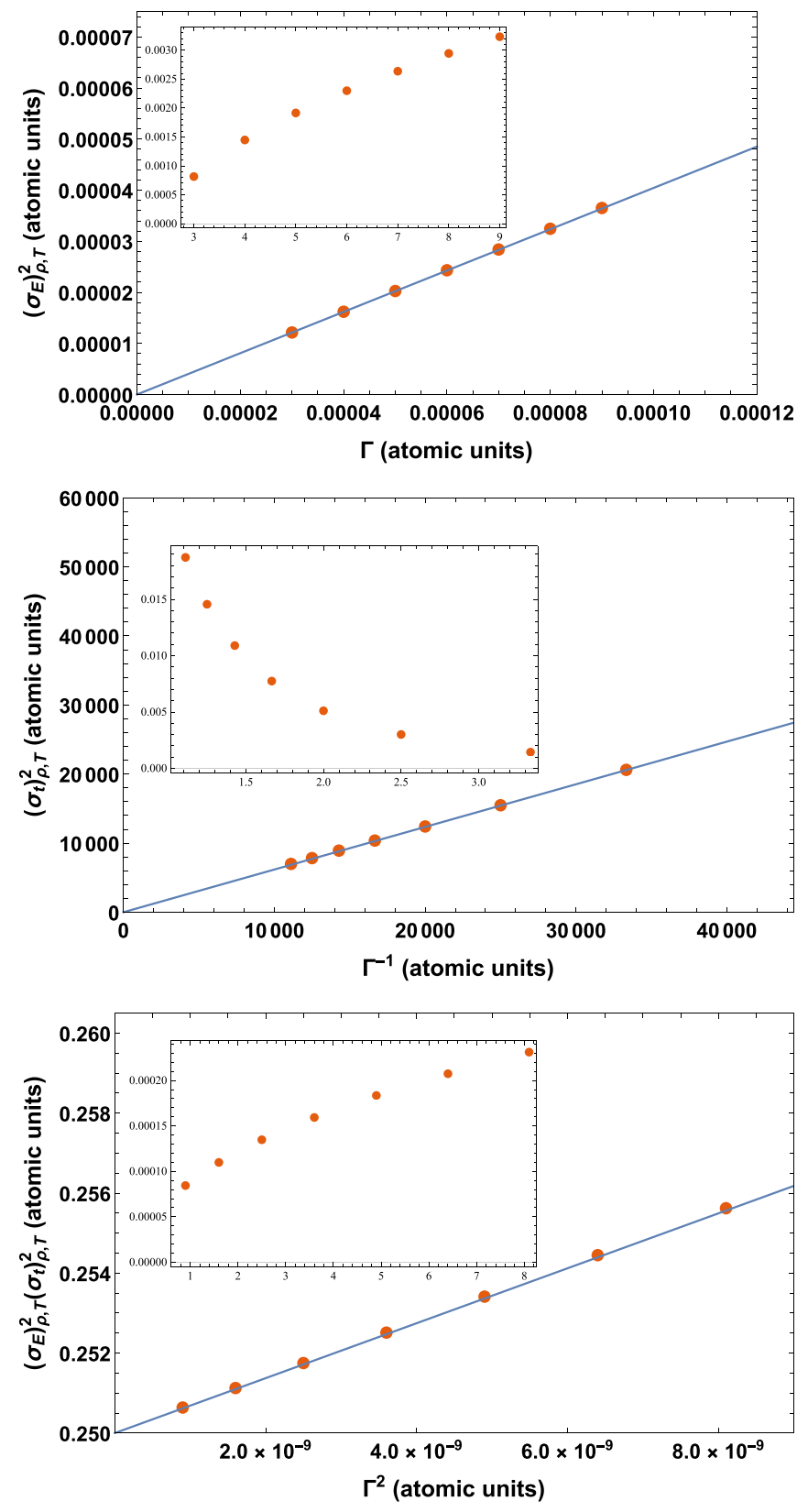

FIG. 4. The variances of the energy $\left(\sigma_{E}\right)^{2}$ and time $\left(\sigma_{t}\right)^{2}$ for $a=$ $5, p_{x}=0.9, V^{\ddagger}=1.5$ for the transmitted density, plotted as functions of $\Gamma$ and $\Gamma^{-1}$, respectively, along with the product of the variances of the two parameters $\left(\sigma_{E}\right)^{2}\left(\sigma_{t}\right)^{2}$ plotted as a function of $\Gamma^{2}$. The points represent the results of the full calculations, and the lines represent fits done with the steepest-descent approximations. Insets: relative error between the steepest-descent and fully quantum calculations (defined in a similar manner to the inset in Fig. 3) for each of the three plots (with the horizontal axes scaled by $10^{5}, 10^{-4}$, and $10^{9}$, respectively).

Table $\mathrm{V}$ do so more clearly. The quantity $t_{\text {trav }}$, as defined in Eq. (13), effectively defines an "outside-the-barrier" traversal time for the transmitted particle by means of a comparison with a free particle, and then subtracts that from the peak time of flight, under the assumption that the leftover represents an "inside-the-barrier" traversal time.
TABLE V. The barrier traversal time of Eq. (13), $t_{\text {trav }}$, of the transmitted wave packet for different values of the barrier width $a$ (with $V^{\ddagger}=1.5$ and $p_{x}=0.9$ ), contrasted with the naive classical free-particle traversal time $\frac{2 a M}{p_{x}}$ and with the two phase times. The quantity $t_{\max , \rho, T}$ is given in the limit $\Gamma \rightarrow 0 . t_{\text {trav }}$ is qualitatively similar for $t_{\max , J, T}, t_{\max , \rho, R}$, and $t_{\max , J, R}$.

\begin{tabular}{lccccl}
\hline \hline $2 a$ & $t_{\max , \rho, T}(a)$ & $\frac{2 a M}{p_{x}}$ & \multicolumn{1}{c}{$\Phi_{T}(a)$} & $\Phi_{R}(a)$ & $t_{\text {trav }}(a)$ \\
\hline 0.2 & 1667.043 & 0.2222222 & 0.3765323 & 0.5987546 & 0.5985556 \\
2.0 & 1665.947 & 2.222222 & -0.7193311 & 1.502891 & 1.502556 \\
6.0 & 1661.502 & 6.666667 & -5.165028 & 1.501639 & 1.502000 \\
10.0 & 1657.056 & 11.11111 & -9.609472 & 1.501639 & 1.500444 \\
\hline \hline
\end{tabular}

In this table, the peak of the density distribution as a function of flight time for the tunneled particle $t_{\max , \rho, T}$ is given for four different values of the barrier width $a$ (keeping the barrier height $V^{\ddagger}$ and incident mean momentum $p_{x}$ constant at the values they had in Fig. 1, and taking the limit $\Gamma \rightarrow 0$ ), and compared to the naive barrier traversal time, the two phase times, and $t_{\text {trav }}$. Results were also obtained for the flux, but are not displayed as they are almost identical to the results for the density, as demonstrated also in other tables.

Three facts can be clearly discerned from the results presented in Table V. The first is that for the square barrier, $t_{\text {trav }}$ is independent of the barrier width $a$, when $a$ is sufficiently large. The second is that the tunneling time $t_{\text {trav }}$ is almost precisely equal to the reflected phase time $\Phi_{R}$, reinforcing the conclusion reached using comparisons to Wigner dynamics. The third is that the constancy of the tunneling time sets in at a length at which the transmitted phase time delay becomes negative. This relationship makes sense when considering Eq. (19) since for the square barrier, $\Phi_{T}$ is the same as $\Phi_{R}$ minus the naive free-particle barrier traversal time.

To further emphasize the fact that the barrier flight time converges to the reflected phase time, Fig. 6 presents $t_{\text {trav }}$ before the $\Gamma \rightarrow 0$ limit is imposed, as a function of $\Gamma$. This barrier traversal time is calculated from both the transmitted and reflected flight times, which both converge to the same quantity $\Phi_{R}$ for $\Gamma \rightarrow 0$, and which overlap on this plot.

The classical Wigner approximations of these numbers were also computed, as seen in Fig. 3 and Table II. Since the results obtained using the classical Wigner approximations presuppose that the tunneling time was zero, the Wigner function equivalent of $t_{\max , \rho, T}$ also deviated from the QM version by almost exactly the phase time $\Phi_{R}(a)$.

While the comparisons to the reflected classical Wigner approximation and to free particles are useful, it would still be desirable to have a measure of the tunneling time that did not rely on comparisons based on classical mechanics, and only used the quantum properties of the transmitted particle itself. As previously mentioned, the mean time for the free-particle wave packet diverges, so it cannot be used as a reference for the mean time.

Therefore, instead of comparing the mean transmission time with a classical Wigner time or comparing peak times with the free particle, one may compare mean transmission times for the same initial wave packet traversing through barriers with the same widths, but different heights. If our 


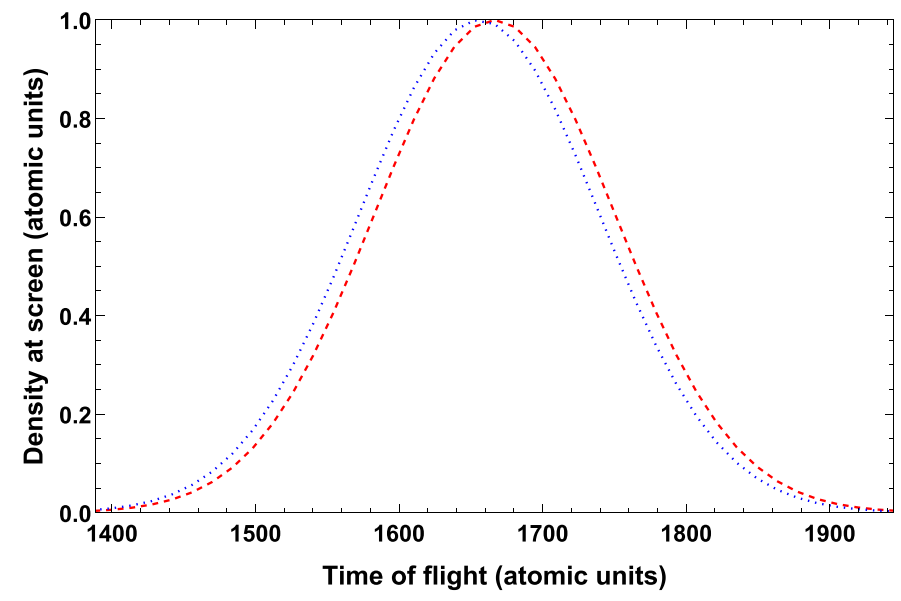

Free Particle Density

(divided by peak density $5.2932 \times 10^{-3}$ )

Tunneled Particle Density

(divided by peak density $2.3481 \times 10^{-15}$ )

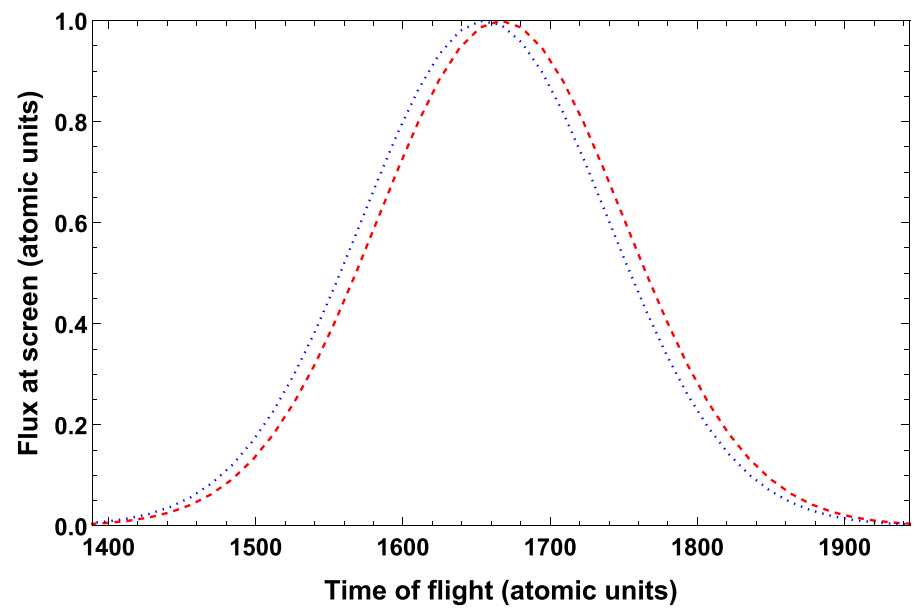

- . - Free Particle Flux

(divided by peak flux $4.7639 \times 10^{-3}$ )

Tunneled Particle Flux

(divided by peak flux $2.1147 \times 10^{-15}$ )

FIG. 5. Free and transmitted particle density and flux distributions, normalized to their respective peak values. The tunneled particle clearly peaks earlier. All parameters are as in Fig. 1.

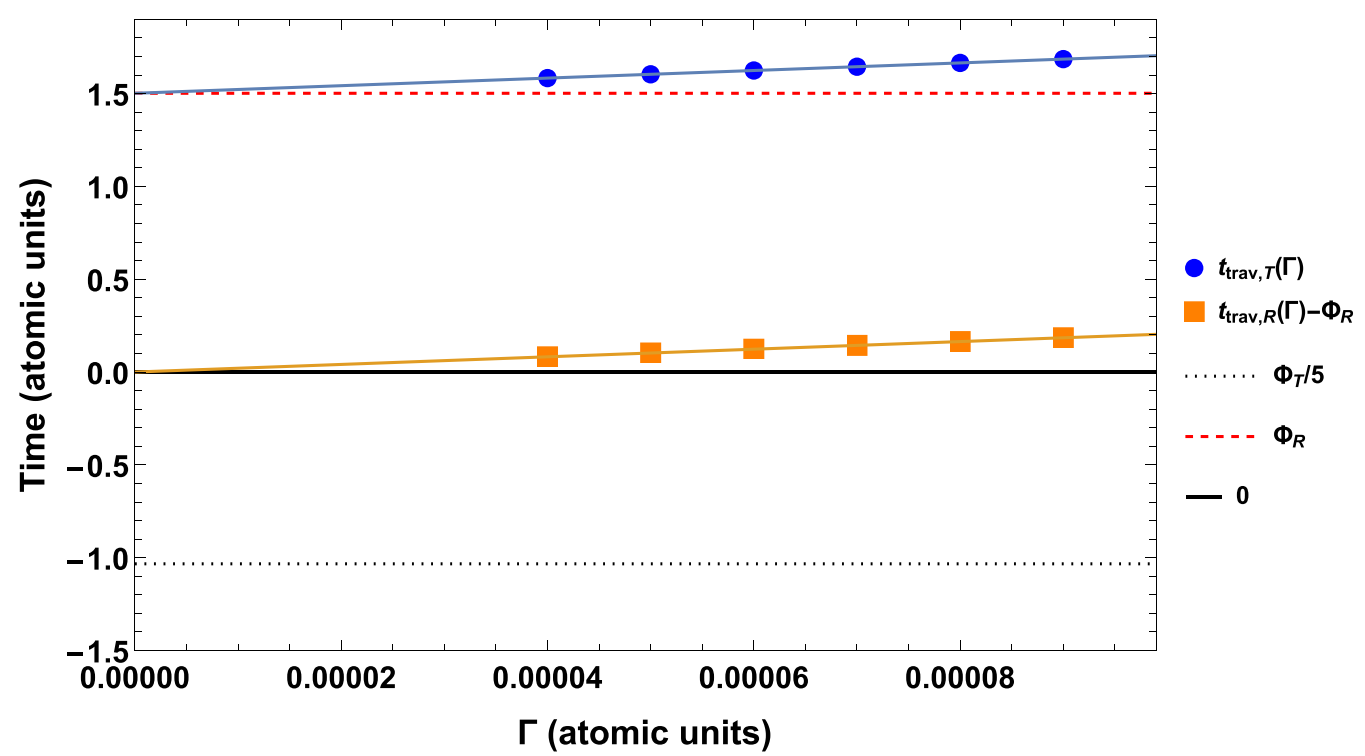

FIG. 6. The barrier tunneling time from Eq. (13) calculated using the mean transmitted (closed circular points and closest line) and reflected (closed square points and closest line) flight times $\left[t_{\text {trav }, T}(\Gamma)\right.$ and $\left.t_{\text {trav }, R}(\Gamma)\right]$ as functions of $\Gamma$ compared to the transmitted and reflected phase times $\Phi_{T}$ and $\Phi_{R}$ for $p_{x}=0.9, V^{\ddagger}=1.5, a=3, x=-500, y=1000$. The quantity $\Phi_{R}$ is subtracted from $t_{\text {trav }, R}(\Gamma)$ to distinguish it from $t_{\text {trav }, T}(\Gamma)$, which it would otherwise overlap with, and $\Phi_{T}$ is divided by five to make the slopes of the fitted lines clearer. 


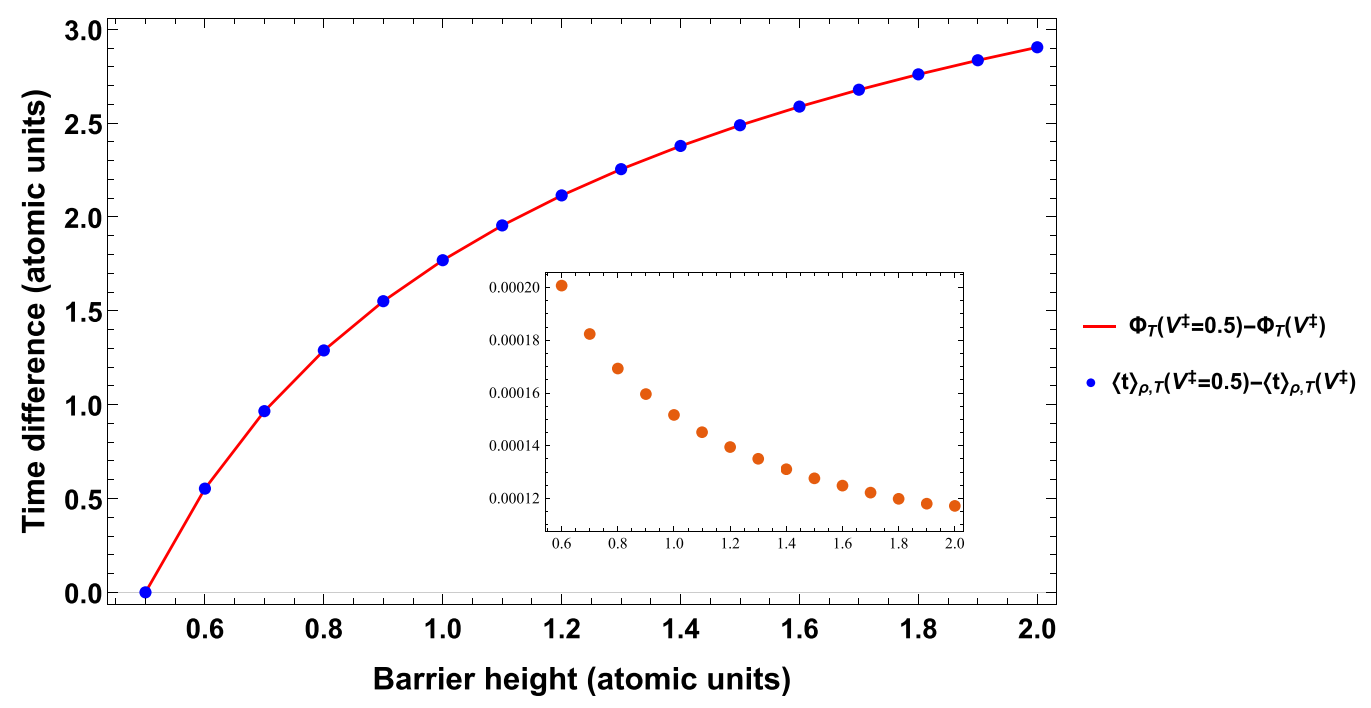

FIG. 7. Mean time of flight for the density of the transmitted particle $\langle t\rangle_{\rho, T}$, subtracted from its value at barrier height $V^{\ddagger}=0.5$, all as a function of barrier height (dots), compared with the transmitted phase time $\Phi_{T}$, also as a function of $V^{\ddagger}$ and subtracted from its value at $V^{\ddagger}=0.5$ (line). The numerical parameters were $a=5, p_{x}=0.4$, and $\Gamma \rightarrow 0$. Inset: the relative error $\sqrt{\frac{\left[\left[\langle t\rangle(0.5)-\langle t\rangle\left(V^{\ddagger}\right)\right]-\left[\Phi_{T}(0.5)-\Phi_{T}\left(V^{\ddagger}\right)\right]\right]^{2}}{\left[\Phi_{T}(0.5)-\Phi_{T}\left(V^{\ddagger}\right)\right]^{2}}}$ between the points and the curve as a function of the barrier height $V^{\ddagger}$.

conclusion that the mean tunneling time is given by the reflected phase time is correct, we should expect that, in the narrow-in-momentum limit, the difference between the flight times for two different barrier heights should be given by the difference between the reflected phase times for the two barrier heights.

To evaluate this, the $\Gamma \rightarrow 0$ mean tunneling time for the density $\langle t\rangle_{\rho, T}$ was considered for different values of the barrier height $V^{\ddagger}$ (once again, flux results were computed but not displayed as they were almost identical to the density results). The lowest value of $V^{\ddagger}, V^{\ddagger}=0.5$, was chosen as a reference value (for no value of $V^{\ddagger}$ in this analysis did the initial momentum distribution have significant above-barrier components). $\langle t\rangle_{\rho, T}$ was evaluated at this value, and then $\langle t\rangle_{\rho, T}\left(V^{\ddagger}\right)$ was subtracted from this quantity for all values of $V^{\ddagger}$ considered. The same principle was then applied to the phase time $\Phi_{T}$, and the results were compared. These results are shown in Fig. 7. Once again, the tunneling time is shown to be directly related to the phase time. As the barrier height increases, the mean time of flight and the phase time decrease in tandem. We anticipate that this last result should also be valid for other types of barrier.

The results shown for $\Phi_{T}$ in Fig. 7 apply equally well to $\Phi_{R}$ since Eq. (19) shows that the difference between the reflected and transmitted phase times does not depend on $V^{\ddagger}$, and thus $\Phi_{T}\left(V^{\ddagger}\right)-\Phi_{T}\left(V^{\ddagger^{\prime}}\right)$ is the same as its reflected counterpart.

\section{DISCUSSION}

\section{A. Special relativity}

The MacColl-Hartman effect presents clear, problematic implications for special relativity: if a long barrier can be traversed in negligible time, then it is trivially simple to construct a way for a quantum object to travel "superluminally." Since the barrier traversal time $t_{\text {trav }}$ is constant, as the barrier width $2 a$ increases, $t_{\text {trav }}$ becomes a smaller fraction of the naive free-particle time, meaning that for sufficiently large $a$ it is negligible, and thus the wave packet can traverse the barrier with arbitrary "speed."

This fact has led to controversies at the borders of quantum mechanics and special relativity over the physical implications of observations made on tunneled systems [5,25,4548]. These controversies persist due to disagreements over the definitions of quantities like the "traversal time." Due to the quantum mechanical nature of the objects and processes involved, there are grounds for disagreement over the definitions that correspond most closely to sensible observables [1,5,15,16,25,49].

Our results demonstrate that there is a causal connection between the wave packet that enters the barrier and the one that exits it, as the two are shown to have the same shape (mainly the amplitude is changed). This is an important insight in the context of claims $[5,25,26,46]$ that the tunneling time metrics are somehow flawed due to the tunneled object being a different entity. We showed that the variances of the reflected and transmitted wave packets are almost identical, a clear sign that the transmitted wave packet has a causal connection to the initial one.

A deeper discussion of all of the disagreements, along with a discussion of the implications of tunneling times in a relativistic framework, are presented in a separate paper [10]. That paper also shows that qualitatively similar results can be obtained when using the Dirac equation, which, with few exceptions [9,50,51], has not been explored thoroughly for this problem.

\section{B. Square barriers}

The results presented in this work show that, for the given definitions and assumptions used to construct a "tunneling 


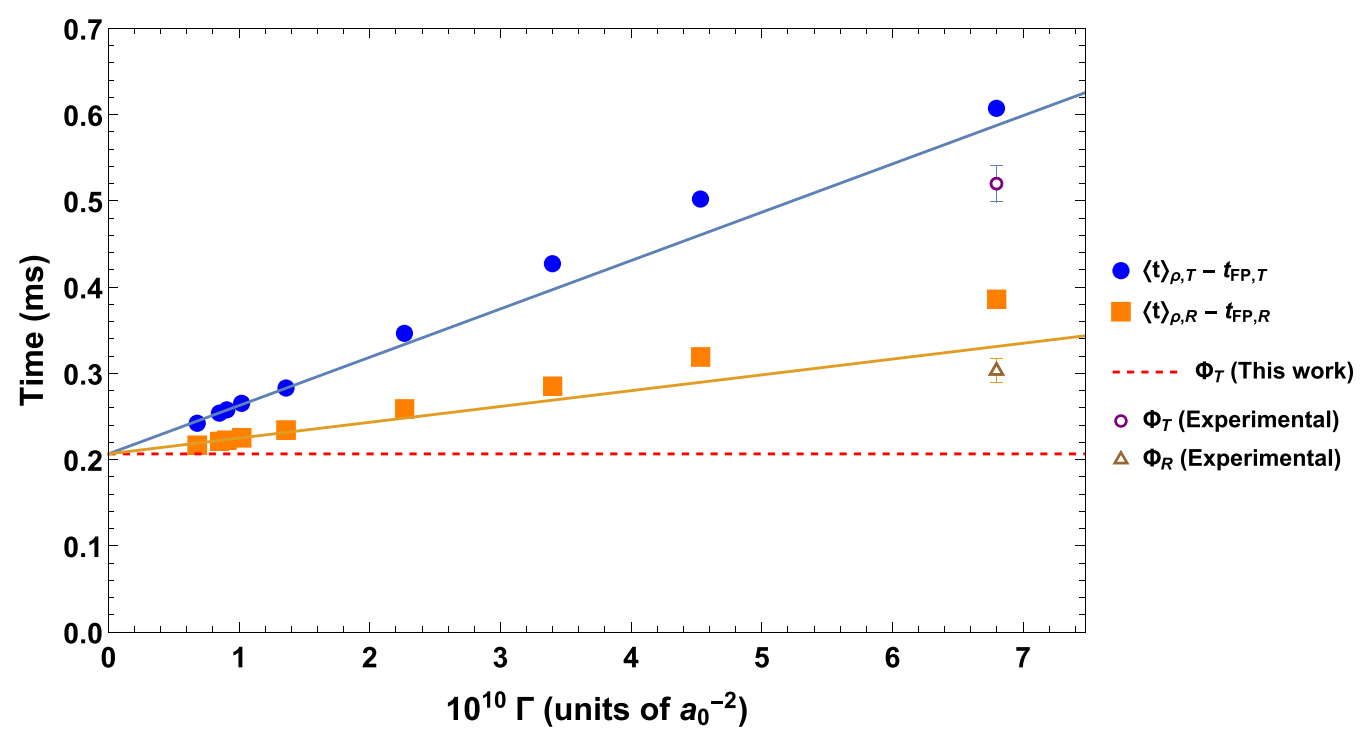

FIG. 8. The mean transmitted time $\langle t\rangle_{\rho, T}$ minus the momentum-filtered free-particle time $t_{\mathrm{FP}, T}$ (closed circular points and closest line), the equivalent points for the reflected component (closed square points and closest line), and the transmitted phase time for a Gaussian barrier $\Phi_{T}$ are all plotted as functions of the incident wave-packet width parameter $\Gamma$ in inverse-square Bohr radii $\left(a_{0}^{-2}\right)$. The lines of best fit are produced using the first five points of each data set, assuming the intercept equals the phase time. The open circular and open triangular points with error bars are the experimentally reported transmitted and reflected times, respectively, at the experimental $\Gamma$ and the same mean incident energy.

time," the time spent under the square barrier is small but finite, and becomes independent of barrier length. Specifically, the time spent under the barrier is equal to $\Phi_{R}$, which is independent of the barrier length for sufficiently long barriers.

This work also shows that the reflected wave packet has the same time distribution as the transmitted wave packet, which suggests that it also "spends some time under the barrier" before being reflected. It was also shown that the flux time distributions are very similar to the density time distributions (apart from the negativity of the reflected flux). From a theoretical perspective, this can be verified using the steepest-descent approximation. The results based on the flux time distributions may be able to motivate experimental works in the future.

It is important to note that the results produced here only demonstrate the MacColl-Hartman effect so vividly because the initial wave packet is carefully constructed to avoid above-barrier transmission and pretransmission. In essence, we verify the MacColl-Hartman effect only for the case of a square barrier, and only for a very small portion of the possible phase space for the preparation of the incident particle.

The square barrier greatly simplifies the task of relating quantum objects to classical counterparts since the dynamics can be understood in terms of prebarrier, under-barrier, and postbarrier components with relative ease. Analysis of other barriers encounters problems assigning those labels since the potential affects the motion in the classically allowed region. In our results, numerical testing showed that any above-barrier transmission or significant pretransmission notably altered the final results, in many cases drowning out the relationship with the phase time. The choice of the incident wave packet can significantly affect the results and mask the tunneling.

\section{Experimental results}

The impact of the choice of incident wave packet on the transmission dynamics is further emphasized by comparing to recent experimental work by Spierings and Steinberg [16] (expanding on results presented by the same group in Ramos et al. [15]) which used the Larmor clock measure [46] of tunneling time. For a symmetric barrier, this is closely related to the phase time [52,53], and should lend itself to direct comparisons in that way.

Figure 8 is similar in construction to Fig. 6 but with important differences. Figure 8 shows the mean transmitted and reflected times for the density of a Gaussian wave packet incident on a Gaussian barrier (calculated with the code wavepacket [54]), using the parameters and one of the below-barrier energies ( $p_{x}=0.00028656$ atomic units) quoted in [16]. We set $x_{i}$ to be -429912 , and $y$ to 859826 atomic units. As with Fig. 6, here we subtract from the mean transmitted and reflected times the free-particle time of flight. Crucially, however, unlike with Fig. 6, there is no need to subtract the extra $\frac{2 a M}{p_{x}}$ term from the free-particle flight time since the reflected and transmitted phase times for the Gaussian barrier are identical. The free-particle time of flight is adjusted to use the filtered average momentum, rather than the initial momentum, which we calculate with a variant on Eq. (B8). The filtered free-particle momentum was used to eliminate the trivial speedup of the transmitted particle, which becomes larger as the "screen" is moved further away from the barrier.

The plot also shows the phase time at the incident mean energy, calculated using methods derived from [55] (for a symmetric Gaussian barrier, the transmitted and reflected phase times are equal). This method also allows us to calculate the transmission and reflection probabilities $|T(k)|^{2}$ and $|R(k)|^{2}$ for a Gaussian barrier. 
The experiment only used one value of $\Gamma$, but we varied it to produce the figure, with the experimental $\Gamma, 6.7965 \times$ $10^{-10}$ atomic units, being the largest one shown in the plot. At this value of $\Gamma$ we also plot the two experimental tunneling times: the upper one is the transmitted point, and the lower one is the reflected point.

The plot also shows lines of best fit made using the lowest five points, which we assumed to be in the linear regime as with previous plots in this paper. The $y$ intercepts of the lines were assumed to be the phase time, and the resulting residuals between the data points and the lines were calculated accordingly. Within the numerical accuracy of the wave-packet propagation method we used, we found that at the $\Gamma=0$ limit the transmitted and reflected time differences coincided with the phase time.

Inspection of the results presented in Fig. 8 is instructive when considering the experimental results. Most evident is the asymmetry between the transmitted and the reflected times. The plot shows that only in the $\Gamma \rightarrow 0$ limit are the two identical.

In contrast to the claim in [16], this asymmetry does not necessarily reflect an asymmetry in the barrier. It may be due only to the finite spatial width of the incident particle. The transmitted particle "feels" the barrier top more than the reflected particle due to momentum filtering, and so is slowed down more. Hence, a second aspect of these results is the observation that the transmitted time in particular does not accurately reflect the phase time, due to the finite width of the wave packet.

Finally, at best the experiment may reveal a transmission time, but not the "tunneling time" per se, since the experimental Larmor time is affected by the motion in the classically allowed region, whose extent depends on the spatial width of the incident wave packet.

\section{CONCLUSIONS}

We showed that for a Gaussian wave packet transmitted through a one-dimensional square barrier with purely belowbarrier transmission and negligible pretransmission, the time spent under the barrier is small but finite, and we identify this time with the reflected phase time.

Generally, for one-dimensional, smooth, symmetric barriers, the reflected and transmitted phase times are identical, but this is not the case for a square barrier. To make a general statement, we note that the tunneling flight time is given by the reflected phase time and this, then, includes all onedimensional symmetric smooth barriers.

We found that the reflected and transmitted wave packets have the same time-of-flight distributions at their respective screens, with both the means and variances being essentially the same. This demonstrates unequivocally that the incident wave packet is not distorted by the tunneling, meaning that wave-packet distortion is not a "good" explanation for the Hartman effect.

Extensive use was made of the $\Gamma \rightarrow 0$ limit, which removed the complications of momentum filtering from the analysis and allowed the transmitted wave packet to be compared to that of a free particle. Using purely quantum mechanical arguments, along with arguments appealing to classical Wigner dynamics, and comparisons to free particles, we presented numerous metrics one can use to measure time spent under the barrier. In all cases, we showed that these metrics indicate that the time spent under the barrier is given by the reflected phase time, and in doing so we provide a theoretical verification of the MacColl-Hartman effect for the system studied.

Although in the monochromatic limit of $\Gamma \rightarrow 0$ the reflected and transmitted times are identical for smooth, symmetric barriers, this symmetry is destroyed when the width parameter $\Gamma$ is finite. The implications of the present results on experimentally measured Larmor clock-based transmission times were considered. Experimentally, we conclude that there remains a way to go before obtaining a definitive determination of the "tunneling time." The experimental conundrum may still be with us for many years.

\section{ACKNOWLEDGMENTS}

This work was supported by Grant No. 408/19 of the Israel Science Foundation.

\section{APPENDIX A: WEAK-VALUE FLUX UNCERTAINTY RELATION}

In this Appendix we outline the derivation of the weakvalue energy-time uncertainty relation when averaging over the flux time distribution rather than the density distribution, which was used in Ref. [28]. For the density distribution, the weak-value-based definitions of the mean $n=1$ and second moment $n=2$ of the time distribution are given as

$$
\left\langle t^{n}(x)\right\rangle=\int_{0}^{\infty} d t t^{n} P(x, t),
$$

where $P(x, t)$ is given by

$$
P(x, t)=\frac{|\langle x \mid \Psi\rangle|^{2}}{\int_{0}^{\infty} d t|\langle x \mid \Psi\rangle|^{2}}
$$

[the denominator is defined to be $N(x)$ ].

This is a specific case of the general definition of the timeaveraged weak values of operators, given by

$$
\langle O(x)\rangle=\int_{0}^{\infty} d t P(x, t) O_{w}(x, t),
$$

and the time average of products of weak values of operators

$$
\left\langle O_{1}(x) O_{2}(x)\right\rangle=\int_{0}^{\infty} d t P(x, t) O_{1 w}(x, t) O_{2 w}(x, t),
$$

where the weak value of the operator $O$ is defined as

$$
O_{w}(x, t)=\frac{\left\langle x|O| \Psi_{t}\right\rangle}{\left\langle x \mid \Psi_{t}\right\rangle}
$$

In [28] it was shown that, for time moments as defined above, and energy moments based on the Hamiltonian $\hat{H}$, as outlined in Eqs. (23)-(25), the time-energy uncertainty principle was satisfied. In particular, for the standard uncertainty 
principle inequality [56]

$$
0 \leqslant \frac{1}{N(x)} \int_{0}^{\infty} d t P(x, t)\left[t-i \lambda H_{w}(x, t)\right]\left[t+i \lambda H_{w}^{*}(x, t)\right],
$$

it was shown that the wave packet satisfied the inequality

$$
\left\langle t^{2}\right\rangle\left\langle H^{*} H\right\rangle \geqslant \frac{\hbar^{2}}{4} .
$$

That analysis gave all the operator moments with respect to the density of the wave packet at the point $x$. If one wishes instead to use the flux, the quantity $P(x, t)$ is replaced with

$$
P_{J}(x, t)=\frac{J(x, t)}{\int_{0}^{\infty} d t J(x, t)}
$$

for the definition of $J(x, t)$ given in Eq. (5) [where the denominator is denoted as $N_{J}(x)$ ], and all the definitions that flow from it must be updated to flux-based versions such as $O_{w, J}$ and $\langle O(x)\rangle_{J}$.

It remains to be shown that the equivalent of the uncertainty principle inequality in Eq. (A7) with $P(x, t) \rightarrow P_{J}(x, t)$ and $H_{w} \rightarrow H_{w, J}$ is still satisfied. This can be done, as long as it is assumed that $\frac{\partial p_{w}}{\partial t}$, the time derivative of the weak-value version of the momentum operator, is zero. This is equivalent to assuming the potential does not vary in space. Since the flux must be measured far away from the interaction region in order to be useful as a POVM, this assumption is valid.

\section{APPENDIX B: STEEPEST-DESCENT APPROXIMATION}

The steepest-descent approximation, applied to the $k$ integral which determines $\psi(q, t)$, together with a second-order Taylor expansion about the most probable tunneling time, determines a Gaussian (in time) approximation for $\psi(q, t)$ at the screen $(q= \pm y)$. This calculation is carried out in Ref. [10], in the relativistic setting, using the Dirac equation. The corresponding derivation, in the nonrelativistic limit, is provided here.

We begin with the expression for the time-evolving wave packet beyond the barrier in the energy representation, written here as an integral over $k$ :

$$
\begin{aligned}
\psi(q, t)= & C \int_{-\infty}^{\infty} d k \exp \left(-\frac{\left(k-k_{\text {init }}\right)^{2}}{2 \Gamma}\right) \\
& \times T(k) \exp \left(i k(q-x)-\frac{i \hbar k^{2} t}{2 m}\right) \\
= & C \int_{-\infty}^{\infty} d k \exp [i F(k, t)],
\end{aligned}
$$

where for $q=y$,

$$
\begin{aligned}
F(k, t)= & k(y-x)-\frac{\hbar k^{2} t}{2 m} \\
& +\varphi_{T}+i\left(\frac{\left(k-k_{\text {init }}\right)^{2}}{2 \Gamma}-\ln [|T(k)|]\right)
\end{aligned}
$$

and $C$ is the initial wave-packet normalization factor. An equivalent expression can be obtained for the reflected wave packet by replacing $T(k)$ and $\varphi_{T}$ with $R(k)$ and $\varphi_{R}$, and replacing $y$ with $-y$. Similarly, an expression for a free particle can be obtained by simply removing the terms containing $T(k)$ and $\varphi_{T}$ from the expression for $F(k, t)$.

Setting $\partial F / \partial k=0$, determines the saddle-point wave number $k^{\sharp}$. Specifically, $k^{\sharp}$ is the solution to the nonlinear equation

$$
y-x-\frac{\hbar k t}{m}+\frac{d \varphi_{T}}{d k}+i\left(\frac{k-k_{\text {init }}}{\Gamma}-\frac{d \ln [|T(k)|]}{d k}\right)=0 .
$$

In the steepest-descent approximation, the wave packet takes the form

$$
\psi_{\mathrm{sd}}(y, t)=C(2 \pi)^{1 / 2}\left(\frac{d^{2} F^{\sharp}}{d k^{2}}\right)^{-1 / 2} \exp \left[i F\left(k^{\sharp}, t\right)\right] .
$$

The most probable time $t_{\mathrm{mp}}$ is the time for which $\operatorname{Im} F\left(k^{\sharp}, t\right)$ is maximal. This time is determined by setting $d \operatorname{Im} F\left(k^{\sharp}, t\right) / d t$ to zero, to get

$$
\begin{aligned}
\frac{d k^{\sharp}}{d t}\left(\frac{\partial \operatorname{Im} F(k, t)}{\partial k}\right)^{\sharp}+\frac{\partial \operatorname{Im} F\left(k^{\sharp}, t\right)}{\partial t} & =0, \\
\frac{\partial \operatorname{Im} F\left(k^{\sharp}, t\right)}{\partial t} & =0 .
\end{aligned}
$$

The first term in the first line of Eq. (B5) is zero, as this is the defining equation of $k^{\sharp}$. Equation (B5) leads to $\operatorname{Im} k^{\sharp}=0$, i.e., $k^{\sharp}$ is real at the most probable time. This simplifies (B3), as the real and imaginary parts of this equation are easily separated when $k^{\sharp}$ is real.

From the real part, we find

$$
y-x=\frac{\hbar k_{\mathrm{mp}}^{\sharp} t_{\mathrm{mp}}}{m}-\left(\frac{d \varphi_{T}}{d k}\right)_{\mathrm{mp}}^{\sharp}
$$

(where the $\sharp$ superscript and mp subscripts represent evaluating the derivative at $k=k_{\mathrm{mp}}^{\sharp}$ ) which determines $t_{\mathrm{mp}}$. Specifically,

$$
t_{\mathrm{mp}}=\left[y-x+\left(\frac{d \varphi_{T}}{d k}\right)_{\mathrm{mp}}^{\sharp}\right] \frac{m}{\hbar k_{\mathrm{mp}}^{\sharp}} .
$$

In the free-particle case, the phase derivative term is no longer present. As expected, the phase leads to a shift in the most probable time.

From the imaginary part, we find

$$
\frac{k_{\mathrm{mp}}^{\sharp}-k_{\text {init }}}{\Gamma}+\left(\frac{d \ln |T|}{d k}\right)_{\mathrm{mp}}^{\sharp}=0
$$

or

$$
k_{\mathrm{mp}}^{\sharp}=k_{\text {init }}-\Gamma\left(\frac{d \ln |T|}{d k}\right)_{\mathrm{mp}}^{\sharp},
$$

which is a real nonlinear equation determining $k_{\mathrm{mp}}^{\sharp}$. This equation shows the momentum-filtering effect. The wave number is shifted to a higher value since $\ln |T|$ decreases with increasing $k$, and the shift is proportional to $\Gamma$. This simplifies to $k_{\mathrm{mp}}^{\sharp}=k_{\text {init }}$ in the free-particle case.

Expanding $F\left(k^{\sharp}, t\right)$ to second order about the most probable tunneling time gives a wave-packet approximation with a 
Gaussian time dependence:

$$
\begin{aligned}
F\left(k^{\sharp}, t\right)= & F\left(k_{\mathrm{mp}}^{\sharp}, t_{\mathrm{mp}}\right)+\frac{\partial F\left(k_{\mathrm{mp}}^{\sharp}, t_{\mathrm{mp}}\right)}{\partial t}\left(t-t_{\mathrm{mp}}\right) \\
& +\frac{1}{2} \frac{\partial^{2} F\left(k_{\mathrm{mp}}^{\sharp}, t_{\mathrm{mp}}\right)}{\partial k^{2}}\left(k^{\sharp}-k_{\mathrm{mp}}^{\sharp}\right)^{2} \\
& +\frac{\partial^{2} F\left(k_{\mathrm{mp}}^{\sharp}, t_{\mathrm{mp}}\right)}{\partial k \partial t}\left(k^{\sharp}-k_{\mathrm{mp}}^{\sharp}\right)\left(t-t_{\mathrm{mp}}\right) .
\end{aligned}
$$

The above equation accounts for the saddle-point condition (B3) and the linear explicit time dependence of $F$, the second partial with respect to time is zero. The equation is further simplified by differentiating Eq. (B3) with respect to time to get

$$
-\frac{\hbar k^{\sharp}}{m}+\frac{d k^{\sharp}}{d t}\left[-\frac{\hbar t}{m}+\frac{d^{2} \varphi_{T}}{d k^{2}}+i\left(\frac{1}{\Gamma}-\frac{d^{2} \ln |T|}{d k^{2}}\right)\right]=0
$$

or

$$
\frac{d k^{\sharp}}{d t}=\frac{v^{\sharp}}{\left[-\frac{\hbar t}{m}+\frac{d^{2} \varphi_{T}}{d k^{2}}+i\left(\frac{1}{\Gamma}-\frac{d^{2} \ln |T|}{d k^{2}}\right)\right]},
$$

where $v^{\sharp}=\hbar k^{\sharp} / m$. The $\left(k^{\sharp}-k_{\mathrm{mp}}^{\sharp}\right)$ factors in the last two terms in Eq. (B9) are eliminated in favor of $\left(t-t_{\mathrm{mp}}\right)$ factors via

$$
\left(k^{\sharp}-k_{\mathrm{mp}}^{\sharp}\right)=\frac{d k^{\sharp}}{d t}\left(t-t_{\mathrm{mp}}\right)
$$

and Eq. (B11).

Since

$$
\begin{aligned}
& \frac{\partial F(k, t)}{\partial t}=-\frac{\hbar k^{2}}{2 m}, \\
& \frac{\partial^{2} F(k, t)}{\partial k \partial t}=-\frac{\hbar k}{m},
\end{aligned}
$$

and

$$
\frac{\partial^{2} F(k, t)}{\partial k^{2}}=-\frac{\hbar t}{m}+\frac{d^{2} \varphi_{T}}{d k^{2}}+i\left(\frac{1}{\Gamma}-\frac{d^{2} \ln |T|}{d k^{2}}\right),
$$

we have

$$
\begin{aligned}
F\left(k^{\sharp}, t\right)= & F\left(k_{\mathrm{mp}}^{\sharp}, t_{\mathrm{mp}}\right)-\frac{\hbar k_{\mathrm{mp}}^{\sharp 2}}{2 m}\left(t-t_{\mathrm{mp}}\right) \\
& -\frac{1}{2} \frac{v_{\mathrm{mp}}^{\sharp 2}}{-\frac{\hbar t}{m}+\left(\frac{d^{2} \varphi_{T}}{d k^{2}}\right)_{\mathrm{mp}}^{\sharp}+i\left[\frac{1}{\Gamma}-\left(\frac{d^{2} \ln |T|}{d k^{2}}\right)_{\mathrm{mp}}^{\sharp}\right]} \\
& \times\left(t-t_{\mathrm{mp}}\right)^{2} .
\end{aligned}
$$

This leads to the following approximate wave packet:

$$
\begin{aligned}
\psi_{\mathrm{sd}}(y, t)= & \left|T_{\psi, \mathrm{sd}}\right| \pi^{-1 / 4}(\operatorname{Re} \eta)^{-1 / 2} \\
& \times \exp \left(-i \frac{\hbar k_{\mathrm{mp}}^{\sharp 2}}{2 m}\left(t-t_{\mathrm{mp}}\right)-\frac{\eta}{2}\left(t-t_{\mathrm{mp}}\right)^{2}\right),
\end{aligned}
$$

where $\left|T_{\psi, \text { sd }}\right|$ is the equivalent of $\left|T_{\psi}\right|$ for the steepest-descent calculation, and

$$
\eta=\frac{v_{\mathrm{mp}}^{\sharp 2}}{\frac{1}{\Gamma}-\left(\frac{d^{2} \ln |T|}{d k^{2}}\right)_{\mathrm{mp}}^{\sharp}-i\left[\left(\frac{d^{2} \varphi_{T}}{d k^{2}}\right)_{\mathrm{mp}}^{\sharp}-\frac{\hbar t_{\mathrm{mp}}}{m}\right]}
$$

determines the width of the Gaussian: $\operatorname{Re} \eta^{-1 / 2}$.
The weak-value energy density-weighted moments are readily found using the steepest-descent approximate wave packet:

$$
\begin{aligned}
\langle E\rangle_{\rho, T}= & \left|T_{\psi, \mathrm{sd}}\right|^{-1} i \hbar \int_{0}^{\infty} d t \psi^{*}(a, t) \frac{\partial}{\partial t} \psi(a, t) \\
= & \pi^{-1 / 2}(\operatorname{Re} \eta)^{1 / 2} \int_{0}^{\infty} d t\left[E_{\mathrm{mp}}^{\sharp}-i \hbar \eta\left(t-t_{\mathrm{mp}}\right)\right] \\
& \times \exp \left[-\operatorname{Re} \eta\left(t-t_{\mathrm{mp}}\right)^{2}\right] \\
\cong & E_{\mathrm{mp}}^{\sharp}
\end{aligned}
$$

and

$$
\begin{aligned}
\left\langle E^{2}\right\rangle_{\rho, T}= & \left|T_{\psi, \mathrm{sd}}\right|^{-1} \hbar^{2} \int_{0}^{\infty} d t \frac{\partial}{\partial t} \psi^{*}(a, t) \frac{\partial}{\partial t} \psi(a, t) \\
= & \pi^{-1 / 2}(\operatorname{Re} \eta)^{1 / 2} \int_{0}^{\infty} d t\left|E_{\mathrm{mp}}^{\sharp}-i \hbar \eta\left(t-t_{\mathrm{mp}}\right)\right|^{2} \\
& \times \exp \left[-\operatorname{Re} \eta\left(t-t_{\mathrm{mp}}\right)^{2}\right] \\
\cong & \pi^{-1 / 2} \int_{-\infty}^{\infty} d t\left\{E_{\mathrm{mp}}^{\sharp 2}+\hbar^{2}\left[\operatorname{Re} \eta+(\operatorname{Im} \eta)^{2}(\operatorname{Re} \eta)^{-1}\right] t^{2}\right\} \\
& \times \exp \left(-t^{2}\right) \\
= & E_{\mathrm{mp}}^{\sharp 2}+\frac{1}{2} \hbar^{2}\left[\operatorname{Re} \eta+(\operatorname{Im} \eta)^{2}(\operatorname{Re} \eta)^{-1}\right] .
\end{aligned}
$$

The variance of the weak-value energy has the form

$$
\sigma_{E, \rho, T}^{2}=\frac{1}{2} \hbar^{2}\left[\operatorname{Re} \eta+(\operatorname{Im} \eta)^{2}(\operatorname{Re} \eta)^{-1}\right]
$$

Since

$$
\sigma_{t, \rho, T}^{2}=\frac{1}{2}(\operatorname{Re} \eta)^{-1}
$$

we find

$$
\sigma_{E, \rho, T}^{2} \sigma_{t, \rho, T}^{2}=\frac{1}{4} \hbar^{2}\left[1+\left(\frac{\operatorname{Im} \eta}{\operatorname{Re} \eta}\right)^{2}\right] .
$$

The real and imaginary parts of $\eta$ can be written as

$$
\operatorname{Re} \eta=v_{\mathrm{mp}}^{\sharp 2} \frac{\frac{1}{\Gamma}-\left(\frac{d^{2} \ln |T|}{d k^{2}}\right)_{\mathrm{mp}}^{\sharp}}{\left[\frac{1}{\Gamma}-\left(\frac{d^{2} \ln |T|}{d k^{2}}\right)_{\mathrm{mp}}^{\sharp}\right]^{2}+\left[\left(\frac{d^{2} \varphi_{T}}{d k^{2}}\right)_{\mathrm{mp}}^{\sharp}-\frac{\hbar t_{\mathrm{mp}}}{m}\right]^{2}}
$$

and

$$
\operatorname{Im} \eta=v_{\mathrm{mp}}^{\sharp 2} \frac{\left(\frac{d^{2} \varphi_{T}}{d k^{2}}\right)_{\mathrm{mp}}^{\sharp}-\frac{\hbar t_{\mathrm{mp}}}{m}}{\left[\frac{1}{\Gamma}-\left(\frac{d^{2} \ln |T|}{d k^{2}}\right)_{\mathrm{mp}}^{\sharp}\right]^{2}+\left[\left(\frac{d^{2} \varphi_{T}}{d k^{2}}\right)_{\mathrm{mp}}^{\sharp}-\frac{\hbar t_{\mathrm{mp}}}{m}\right]^{2}} .
$$

Thus, for small $\Gamma$, they have the following leading terms:

$$
\operatorname{Re} \eta \sim \Gamma v_{\mathrm{mp}}^{\sharp 2}
$$

and

$$
\operatorname{Im} \eta \sim \Gamma^{2} v_{\mathrm{mp}}^{\sharp 2}\left[\left(\frac{d^{2} \varphi_{T}}{d k^{2}}\right)_{\mathrm{mp}}^{\sharp}-\frac{\hbar t_{\mathrm{mp}}}{m}\right] .
$$

Therefore, for small $\Gamma$,

$$
\begin{aligned}
& \sigma_{E, \rho, T}^{2} \sim \frac{1}{2} \hbar^{2} \Gamma v_{\mathrm{mp}}^{\sharp 2}, \\
& \sigma_{t, \rho, T}^{2} \sim \frac{1}{2} \Gamma^{-1} v_{\mathrm{mp}}^{\sharp-2},
\end{aligned}
$$


and

$$
\sigma_{E, \rho, T}^{2} \sigma_{t, \rho, T}^{2} \sim \frac{1}{4} \hbar^{2}\left\{1+\Gamma^{2}\left[\left(\frac{d^{2} \varphi_{T}}{d k^{2}}\right)_{\mathrm{mp}}^{\#}-\frac{\hbar t_{\mathrm{mp}}}{m}\right]^{2}\right\} .
$$

These three expressions simplify in the free-particle case since $v_{\mathrm{mp}}^{\sharp}=\frac{\hbar}{m} k_{\text {init }}$, and the derivative term in the final expression vanishes.
The flux-weighted moments are related to the densityweighted moments in a simple way in the steepest-descent model. Specifically,

$$
J(q, t)=\frac{\hbar k_{\mathrm{mp}}^{\sharp}}{m} \rho(q, t) .
$$

The extra velocity factor cancels when the moments are calculated as above, and the final result still applies.
[1] E. Hauge and J. Støvneng, Rev. Mod. Phys. 61, 917 (1989).

[2] R. Landauer, Ber. Bunsen-Ges. Phys. Chem. 95, 404 (1991).

[3] S. Brouard, R. Sala, and J. G. Muga, Phys. Rev. A 49, 4312 (1994).

[4] C. R. McDonald, G. Orlando, G. Vampa, and T. Brabec, J. Phys.: Conf. Ser. 594, 012019 (2015).

[5] H. G. Winful, Phys. Rep. 436, 1 (2006).

[6] T. E. Hartman, J. Appl. Phys. 33, 3427 (1962).

[7] J. G. Muga, in Time in Quantum Mechanics, edited by J. Muga, R. S. Mayato, and Í. Egusquiza (Springer, Berlin, 2008), pp. 31-72.

[8] C. R. Leavens and G. C. Aers, Phys. Rev. B 40, 5387 (1989).

[9] S. De Leo and P. P. Rotelli, Eur. Phys. J. C 51, 241 (2007).

[10] R. S. Dumont, T. Rivlin, and E. Pollak, New J. Phys. 22, 093060 (2020).

[11] A. M. Steinberg, P. G. Kwiat, and R. Y. Chiao, Phys. Rev. Lett. 71, 708 (1993).

[12] C. Spielmann, R. Szipöcs, A. Stingl, and F. Krausz, Phys. Rev. Lett. 73, 2308 (1994).

[13] S. Longhi, M. Marano, P. Laporta, and M. Belmonte, Phys. Rev. E 64, 055602(R) (2001).

[14] A. S. Landsman, M. Weger, J. Maurer, R. Boge, A. Ludwig, S. Heuser, C. Cirelli, L. Gallmann, and U. Keller, Optica 1, 343 (2014).

[15] R. Ramos, D. Spierings, I. Racicot, and A. M. Steinberg, Nature (London) 583, 529 (2020).

[16] D. C. Spierings and A. M. Steinberg, Optical, Opto-Atomic, and Entanglement-Enhanced Precision Metrology II, Vol. 11296 (International Society for Optics and Photonics, Bellingham, WA, 2020), p. 112960F.

[17] A. S. Landsman and U. Keller, Phys. Rep. 547, 1 (2015).

[18] L. Torlina, F. Morales, J. Kaushal, I. Ivanov, A. Kheifets, A. Zielinski, A. Scrinzi, H. G. Muller, S. Sukiasyan, M. Ivanov et al., Nat. Phys. 11, 503 (2015).

[19] U. S. Sainadh, H. Xu, X. Wang, A. Atia-Tul-Noor, W. C. Wallace, N. Douguet, A. Bray, I. Ivanov, K. Bartschat, A. Kheifets et al., Nature (London) 568, 75 (2019).

[20] S. U. Sainadh, R. T. Sang, and I. V. Litvinyuk, J. Phys.: Photonics 2, 042002 (2020).

[21] E. A. Galapon, Phys. Rev. Lett. 108, 170402 (2012).

[22] D. A. L. Pablico and E. A. Galapon, Phys. Rev. A 101, 022103 (2020).

[23] L. MacColl, Phys. Rev. 40, 621 (1932).

[24] H. G. Winful, New J. Phys. 8, 101 (2006).
[25] G. Diener, Phys. Lett. A 223, 327 (1996).

[26] G. Nimtz, Prog. Quantum Electron. 27, 417 (2003).

[27] J. Petersen and E. Pollak, J. Phys. Chem. Lett. 8, 4017 (2017).

[28] E. Pollak and S. Miret-Artés, New J. Phys. 20, 073016 (2018).

[29] M. Büttiker and R. Landauer, Phys. Rev. Lett. 49, 1739 (1982).

[30] R. Landauer, Nature (London) 341, 567 (1989).

[31] R. S. Dumont and T. L. Marchioro II, Phys. Rev. A 47, 85 (1993).

[32] J. Petersen and E. Pollak, J. Chem. Phys. 148, 074111 (2018).

[33] G. J. Papadopoulos, Task Quart. 19, 65 (2015).

[34] E. Pollak, Phys. Rev. Lett. 118, 070401 (2017).

[35] J. G. Muga and C. R. Leavens, Phys. Rep. 338, 353 (2000).

[36] I. L. Egusquiza, J. G. Muga, and A. D. Baute, in Time in Quantum Mechanics, edited by J. Muga, R. S. Mayato, and Í. Egusquiza (Springer, Berlin, 2008), pp. 305-332.

[37] S. De Leo, J. Phys. A: Math. Theor. 46, 155306 (2013).

[38] C. Texier, Phys. E (Amsterdam) 82, 16 (2016).

[39] E. Wigner, Phys. Rev. 40, 749 (1932).

[40] E. J. Heller, J. Chem. Phys. 65, 1289 (1976).

[41] J. Muga, S. Brouard, and R. Sala, Phys. Lett. A 167, 24 (1992)

[42] W. B. Case, Am. J. Phys. 76, 937 (2008).

[43] V. Olkhovsky, Adv. Math. Phys. 2009, 859710 (2009).

[44] H. Bauke and N. R. Itzhak, arXiv:1101.2683.

[45] R. Y. Chiao and A. M. Steinberg, Progress in Optics, Vol. 37 (Elsevier, Amsterdam, 1997), pp. 345-405.

[46] D. Sokolovski, in Time in Quantum Mechanics, edited by J. Muga, R. S. Mayato, and Í. Egusquiza (Springer, Berlin, 2008), pp. 195-233.

[47] C. Anastopoulos and N. Savvidou, Ann. Phys. 336, 281 (2013).

[48] G. Nimtz and H. Aichmann, Z. Naturforsch. A 72, 881 (2017).

[49] C-F. Li and X. Chen, Ann. Phys. 11, 916 (2002).

[50] J. T. Lunardi and L. A. Manzoni, Phys. Rev. A 76, 042111 (2007).

[51] I. A. Ivanov and K. T. Kim, Phys. Rev. A 92, 053418 (2015).

[52] H. G. Winful, Phys. Rev. Lett. 91, 260401 (2003).

[53] R. S. Mayato, D. Alonso, and I. L. Egusquiza, in Time in Quantum Mechanics, edited by J. Muga, R. S. Mayato, and Í. Egusquiza (Springer, Berlin, 2008), pp. 235-278.

[54] C. M. Dion, A. Hashemloo, and G. Rahali, Comput. Phys. Commun. 185, 407 (2014).

[55] F. M. Fernández, Am. J. Phys. 79, 877 (2011).

[56] H. P. Robertson, Phys. Rev. 34, 163 (1929). 